\title{
The analytical interface coupling of arbitrary-order fractional nonlinear hyperbolic scalar conservation laws
}

\author{
S.M.R. Shirkhorshidi ${ }^{1}$, W.A.M. Othman² ${ }^{2}$ (D, M.A. Omar Awang ${ }^{2}$, D. Rostamy ${ }^{3}$ and A.S. Shirkhorshidi ${ }^{4}$
}

"Correspondence:

wanainun@um.edu.my ${ }^{2}$ Institute of Mathematical Sciences,

Faculty of Science, University of Malaya, Kuala Lumpur, Malaysia Full list of author information is available at the end of the article

\section{Springer}

\begin{abstract}
In this paper, the existence and uniqueness of the interface coupling (IC) of time and spatial (TS) arbitrary-order fractional (AOF) nonlinear hyperbolic scalar conservation laws (NHSCL) are investigated. The technique of arbitrary fractional characteristic method (AFCM) is used to accomplish this task. We apply Jumarie's modification of Riemann-Liouville and Liouville-Caputo's definition to extend some formulae to the arbitrary-order fractional calculus. Then these formulae are utilized to prove the main theorem. In this process, we develop an analytic method, which gives us the ability to find the solution of IC AOF NHSCL. The feature of this method is that it enables us to verify that the obtained solution satisfies the fractional partial differential equation (FPDE), and the solution is unique. Furthermore, a few examples illustrate the implementation of this technique in the application section.
\end{abstract}

Keywords: Arbitrary-order fractional calculus; Interface coupling arbitrary-order fractional nonlinear scalar conservation law; Arbitrary-order fractional characteristic method; Jumarie's modification of Riemann-Liouville arbitrary-order fractional derivative

\section{Introduction}

The notion of hyperbolic conservation laws (HCLs) was raised about five decades ago $[1,2]$. Long ago, the properties for a partial differential equations' (PDEs') system of this type were distinguished. Moreover, the interface coupling (IC) of HCLs has important applications. Several phenomena occur in mathematical physics, and their mathematical models are expressed in the form of the IC HCLs. Hence many researchers have tried to develop new techniques to find analytical and numerical solutions for IC HCLs. Many of them have been successful in introducing methods to find numerical solutions.

The analytical method has become a very appealing tool to pursue a solution to differential equations (DEs), which leads to the exact answer. Analytical results for most IC FDEs cannot be obtained, so developing a new method is important. To the best of our knowledge, the analytical solution of interface coupling fractional conservation law has not been addressed, yet. This article aims at fulfilling this gap and investigates the analytical solution of IC HCL. In the present paper, we adopt the fractional characteristic

(c) The Author(s) 2020. This article is licensed under a Creative Commons Attribution 4.0 International License, which permits use sharing, adaptation, distribution and reproduction in any medium or format, as long as you give appropriate credit to the original author(s) and the source, provide a link to the Creative Commons licence, and indicate if changes were made. The images or other third party material in this article are included in the article's Creative Commons licence, unless indicated otherwise in a credit line to the material. If material is not included in the article's Creative Commons licence and your intended use is not permitted by statutory regulation or exceeds the permitted use, you will need to obtain permission directly from the copyright holder. To view a copy of this licence, visit http://creativecommons.org/licenses/by/4.0/. 
method (FCM), which is a very powerful technique that converts an IC FPDE to a system of IC FODE, which makes it possible to solve the problem. The FCM method was introduced by Guo-chang Wu [2], and it is further developed to address the IC AOF hyperbolic conservation laws. Due to its efficiency in obtaining the exact solution, it became a very attractive method for seeking answers to differential equations. The feature of this technique compared to the other analytical solution is the ability to check if the obtained solution is a correct answer to our problem by substituting the answer in the IC FPDE and showing it satisfies the differential equation.

The homogeneous interface coupling hyperbolic conservation laws in the form of arbitrary-order fractional (AOF) refers to first-order systems of fractional PDEs in divergence form,

$$
\begin{aligned}
& \begin{cases}\partial_{t}^{\alpha(\tau)} \mathcal{H}(u)+\sum_{i=1}^{m} \mathfrak{F}_{i L}(u) \partial_{x_{i}}^{\beta_{i}(\tau)} \mathcal{G}_{i}(u)=0, & x_{i}<0, t>0, i=1, \ldots, m, \\
\partial_{t}^{\gamma(\tau)} \mathcal{H}(u)+\sum_{i=1}^{m} \mathfrak{F}_{i R}(u) \partial_{x_{i}}^{\lambda_{i}(\tau)} \mathcal{G}_{i}(u)=0, & x_{i}>0, t>0, i=1, \ldots, m,\end{cases} \\
& 0<\alpha(\tau), \gamma(\tau), \beta_{i}(\tau), \lambda_{i}(\tau) \leq 1, \quad \tau \in \mathbb{R},
\end{aligned}
$$

where $u$ is a function of the spatial variables $\left(x_{1}, \ldots, x_{m}\right)$ and time $t$. The given functions $\mathcal{H}, \mathfrak{F}_{i R}, \mathfrak{F}_{i L}$ and $\mathcal{G}_{i}$ where $i=1, \ldots, m$ are smooth maps from $\mathbb{R}$ to $\mathbb{R}$. Also $\alpha: \mathbb{R} \rightarrow(0,1)$, $\gamma: \mathbb{R} \rightarrow(0,1), \beta_{i}(\tau): \mathbb{R} \rightarrow(0,1)$ and $\lambda_{i}(\tau): \mathbb{R} \rightarrow(0,1)$ where $\alpha(\tau), \gamma(\tau), \beta_{i}(\tau)$ and $\lambda_{i}(\tau)$ for $i=1, \ldots, m$ are continuous. The symbol $\partial_{t}$ stands for $\frac{\partial}{\partial t}$, and $\partial_{x}$ stands for $\frac{\partial}{\partial x}$. If we assume $\alpha(\tau), \gamma(\tau)=1$ and $\beta_{i}(\tau), \lambda_{i}(\tau)=1$ for $i=1, \ldots, m$ in the above problem, then it reduces to the classical interface coupling conservation law, numerical approximations of which have been studied by researchers. We are wondering if there is any solution that is unique and satisfies the equation of IC AOF HNCL.

This paper is organized as follows: Sect. 2 elaborates the background on fractional calculus, constant- and variable-order fractional derivative, and preliminaries on the definitions of Riemann-Liouville fractional derivative of variable-order, and Caputo derivative of variable-order are introduced. Then the definitions of Riemann-Liouville fractional derivative of arbitrary-order and Liouville-Caputo derivative of arbitrary-order are presented. Furthermore, the generalization of some integer calculus (IC) formulae to constant- and arbitrary-order calculus, which will be used in later sections, is introduced. Section 3 presents the proof of the existence and uniqueness of IC VOF NHSCL, and in this process, we introduce a very powerful technique to solve interface coupling FPDEs. Section 4 shows the implementation of this analytical method to solve a few physical examples and presents a benchmark for solution of each one. Then, we used MATLAB to sketch the graphs of the obtained solutions.

\section{Literature review}

\subsection{Background}

Partial differential equations (PDEs) are one of the most essential and powerful mathematical tools to describe many phenomena. Scientists have been implementing PDEs in mathematical physics, engineering fields, solid-state physics, quantum mechanics, plasma physics, fluid mechanics, chemical kinematics, ecology, optical fibers, geochemistry, biology, meteorology, and so on. Hence many researchers have tried to develop new techniques to find exact or analytical solutions for PDEs. Many of them have been successful 
in introducing methods to find exact or analytical solutions for PDEs, such as the sinecosine function method and Bernoulli's equation approach [3, 4], the Kudryashov method [5], the functional variable method [6], the $\left(G^{\prime} / G\right)$-expansion method $[7,8]$, Hirota's bilinear method [9], the first integral method [10], etc. However, the integer calculus often contradicts the experimental results, so it is more suitable to use fractional calculus, a generalization of it.

Fractional calculus (FC) exhibited a remarkable evolution during the last three decades and has attracted the attention of many researchers in many scientific areas [11-16]. The definition and concept of fractional derivative (FD) and fractional integral (FI) are presented in different forms. One of them is the Riemann-Liouville derivative [17], which has been mostly used in mathematical framework studies. Still, in the last decade, the Caputo derivative [18] became popular in applied sciences due to the way it is dealing with the initial conditions [18]. Also, the Grünwald-Letnikov definition is considered mostly for approximations in numerical methods. Also, Kilbas et al. [19] have a book titled "Theory and Applications of Fractional Differential Equations."

Samko and Ross [20] introduced the concept of variable-order fractional (VOF) derivative and integral (which is a generalization of constant-order fractional derivative and integral) together with some basic properties in 1993. Lorenzo and Hartley [21] investigated the definitions of VOF operators in different forms and then summarized the research results of the VOF operators. Then, a new extension and valuable implementation of the VOF differential equations' (DEs) models have been further developed [22]. Subsequently, VOF DEs have attracted more and more attention, describing their suitability in modeling, along with a large variety of phenomena, ranging from science to engineering. In particular, we mention anomalous diffusion [23, 24], viscoelastic mechanics [22, 25], the control system [26], chaotic systems [27], petroleum engineering [28], and many other branches of physics and engineering, to name a few [29-33].

The exact solutions of most VOF PDEs cannot be found easily, so numerical methods $[34,35]$ must be used. The solutions of the VOF PDEs are investigated by many authors using powerful numerical techniques. Several numerical methods, such as the homotopy perturbation method [36], the Adomian decomposition method [37], the variational iteration method [38], the differential transform method [39], the fractional Riccati expansion method [40], and the fractional subequation method [41-44], have been suggested for solving FDEs. However, solutions obtained through all these methods are local, and it is important to explore other techniques to find exact analytical solutions of FDEs [45].

\subsection{Preliminaries}

\subsubsection{Riemann-Liouville and Liouville-Caputo variable-order fractional derivative for the function of one variable}

The generalization of the Riemann-Liouville and Liouville-Caputo (LC) derivative from constant to variable-order of differentiation and integration have been presented [46]. Given $\alpha(t) \in(0,1)$, the left and right Riemann-Liouville and LC fractional derivatives and integrals of order $\alpha(t)$ of a function $x:[a, b] \rightarrow \mathbb{R}$ are generally defined in two different ways for Riemann-Liouville and three different ways for LC. The definition of the first type for Riemann-Liouville and of the third type for LC derivatives are presented as follows. 
Definition 1 (Type I Riemann-Liouville fractional derivatives of variable-order $\alpha(t)$ [46]) Given a function $x:[a, b] \rightarrow \mathbb{R}$ and $0<\alpha(t)<1$,

1. Type I left Riemann-Liouville fractional derivative of variable-order $\alpha(t)$ is defined by

$$
{ }_{a} D_{t}^{\alpha(t)} x(t)=\frac{1}{\Gamma[1-\alpha(t)]} \frac{d}{d t} \int_{a}^{t}(t-\tau)^{-\alpha(t)} x(\tau) d \tau
$$

2. Type I right Riemann-Liouville fractional derivative of variable-order $\alpha(t)$ is defined by

$$
{ }_{t} D_{b}^{\alpha(t)} x(t)=\frac{1}{\Gamma[1-\alpha(t)]} \frac{d}{d t} \int_{t}^{b}(\tau-t)^{-\alpha(t)} x(\tau) d \tau .
$$

Definition 2 (Type III Caputo fractional derivatives of variable-order $\alpha(t)$ [46]) Given a function $x:[a, b] \rightarrow \mathbb{R}$ and $0<\alpha(t)<1$,

1. Type III left Caputo derivative of variable-order $\alpha(t)$ is defined by

$$
{ }_{a}^{C} D_{t}^{\alpha(t)} x(t)=\frac{1}{\Gamma[1-\alpha(t)]} \int_{a}^{t}(t-\tau)^{-\alpha(t)} x^{\prime}(\tau) d \tau ;
$$

2. Type III right Caputo derivative of variable-order $\alpha(t)$ is defined by

$$
{ }_{t}^{C} D_{b}^{\alpha(t)} x(t)=\frac{1}{\Gamma[1-\alpha(t)]} \int_{t}^{b}(\tau-t)^{-\alpha(t)} x^{\prime}(\tau) d \tau .
$$

\subsubsection{New definition for Riemann-Liouville and Liouville-Caputo fractional arbitrary-order derivative}

In the above definitions, the variable $t$ in $\alpha(t)$ and $x(t)$ is the same; however, it produces different definitions. Now we would like to introduce a definition where the variables of $\alpha$ and $x$ are different; in this case, we will have only one definition for each, which is proper to name them as the Riemann-Liouville and Liouville-Caputo fractional arbitrary-order derivatives.

Definition 3 (New Riemann-Liouville fractional derivatives of arbitrary-order $\alpha(t)$ ) Given a function $f:[a, b] \rightarrow \mathbb{R}$, and $\alpha: \mathbb{R} \rightarrow(0,1)$, where $f(x)$ and $\alpha(t)$ are continuous,

1. New left Riemann-Liouville fractional derivative of arbitrary-order $\alpha(t)$ is defined by

$$
{ }_{a} D_{x}^{\alpha(t)} f(x)=\frac{1}{\Gamma[1-\alpha(t)]} \frac{d}{d x} \int_{a}^{x}(x-s)^{-\alpha(t)} f(s) d s ;
$$

2. New right Riemann-Liouville fractional derivative of arbitrary-order $\alpha(t)$ is defined by

$$
{ }_{x} D_{b}^{\alpha(t)} f(x)=\frac{1}{\Gamma[1-\alpha(t)]} \frac{d}{d x} \int_{x}^{b}(\mathrm{~s}-x)^{-\alpha(t)} f(s) d s .
$$

Definition 4 (New Liouville-Caputo fractional derivatives of arbitrary-order $\alpha(t)$ ) Given a function $f:[a, b] \rightarrow \mathbb{R}$, and $\alpha: \mathbb{R} \rightarrow(0,1)$, where $f(x)$ and $\alpha(t)$ are continuous, then 
1. New left Liouville-Caputo derivative of arbitrary-order $\alpha(t)$ is defined by

$$
{ }_{a}^{C} D_{x}^{\alpha(t)} f(x)=\frac{1}{\Gamma[1-\alpha(t)]} \int_{a}^{x}(x-\tau)^{-\alpha(t)} f^{\prime}(\tau) d \tau
$$

2. New right Liouville-Caputo derivative of arbitrary-order $\alpha(t)$ is defined by

$$
{ }_{x}^{C} D_{b}^{\alpha(t)} f(x)=\frac{1}{\Gamma[1-\alpha(t)]} \int_{x}^{b}(\tau-x)^{-\alpha(t)} f^{\prime}(\tau) d \tau .
$$

\subsubsection{Some results based on Definitions 3 and 4}

We can extend all the results in Jumarie's paper [47] about the fractional constant-order to the fractional arbitrary-order, based on Definitions 3 and 4 and replacing of $\alpha$ with $\alpha(t)$. Consequently, we present some of these results as follows.

Proposition 1 The following formulas hold true:

$$
\begin{aligned}
& D_{x}^{\alpha(t)} x^{\gamma}=\frac{\Gamma(\gamma+1)}{\Gamma[\gamma+1-\alpha(t)]} x^{\gamma-\alpha(t)}, \quad \gamma>0, \\
& D_{x}^{\alpha(t)} x^{\alpha(t)}=\Gamma[\alpha(t)+1], \\
& D_{x}^{\alpha(t)}\left(e^{\alpha^{\alpha(t)}}\right)=\Gamma[1+\alpha(t)] e^{x^{\alpha(t)}}, \\
& D_{x}^{\alpha(t)} \ln x^{\alpha(t)}=\frac{\Gamma[1+\alpha(t)]}{x^{\alpha(t)}} .
\end{aligned}
$$

The formulae that are presented above will be used to prove the theorem and to solve the examples in the application section. We can also derive the following integrating formulae using (2.10), (2.11), and (2.12).

Proposition 2 The following formulas hold true:

$$
\begin{aligned}
& \int(d x)^{\alpha(t)}=x^{\alpha(t)}, \\
& \int e^{x^{\alpha(t)}}(d x)^{\alpha(t)}=e^{x^{\alpha(t)},} \\
& \int \frac{(d x)^{\alpha(t)}}{x^{\alpha(t)}}=\ln x^{\alpha(t)} .
\end{aligned}
$$

Remark 1 The main tool that we use to prove the theorem is the extension of "the fractional characteristic method" from constant-order to arbitrary-order, introduced by Guocheng Wu [48].

\section{Existence and uniqueness}

Let us consider the IC AOF NHSCL in one dimension which is defined by

$$
\begin{aligned}
& \begin{cases}\partial_{t}^{\alpha(\tau)} u(x, t)+C_{L}(u(x, t)) \partial_{x}^{\beta(\tau)} u(x, t)=0, & x<0, t>0, \\
\partial_{t}^{\gamma(\tau)} u(x, t)+C_{R}(u(x, t)) \partial_{x}^{\lambda(\tau)} u(x, t)=0, & x>0, t>0,\end{cases} \\
& 0<\alpha(\tau), \beta(\tau), \gamma(\tau), \lambda(\tau) \leq 1, \quad \tau \in \mathbb{R} .
\end{aligned}
$$

Let $C_{R}: \mathbb{R} \rightarrow \mathbb{R}$ and $C_{L}: \mathbb{R} \rightarrow \mathbb{R}$ be two "smooth" functions. 
The modified Riemann-Liouville derivative of AOF with parameters $\alpha(\tau)$ and $\beta(\tau)$ is

$$
\begin{aligned}
& { }_{0} D_{y}^{\gamma(\tau)} M(y)=\frac{1}{\Gamma(1-\gamma(\tau))} \frac{d}{d y} \int_{0}^{y}(y-\xi)^{-\gamma(\tau)}(M(\xi)-M(0)) d \xi, \\
& 0<\gamma(\tau) \leq 1, \quad \gamma(\tau):=\alpha(\tau), \beta(\tau), \quad y:=x, t .
\end{aligned}
$$

In equation (3.1), we use the notations $\partial_{t}^{\alpha(\tau)} u(x, t):={ }_{0} D_{t}^{\alpha(\tau)} u(x, t)$ for $0<\alpha(\tau) \leq 1$, and similarly $\partial_{x}^{\beta(\tau)} u(x, t):={ }_{0} D_{x}^{\beta(\tau)} u(x, t)$ for $0<\beta(\tau) \leq 1$.

Theorem 1 Let us consider

$$
\begin{aligned}
& \begin{cases}\partial_{t}^{\alpha(\tau)} u(x, t)+C_{L}(u(x, t)) \partial_{x}^{\beta(\tau)} u(x, t)=0, & x<0, t>0, \\
\partial_{t}^{\gamma(\tau)} u(x, t)+C_{R}(u(x, t)) \partial_{x}^{\lambda(\tau)} u(x, t)=0, & x>0, t>0,\end{cases} \\
& 0<\alpha(\tau), \beta(\tau), \gamma(\tau), \lambda(\tau) \leq 1 \\
& u(x, 0)=f(x), \quad x \in \mathbb{R},
\end{aligned}
$$

with an initial condition $u(x, 0): \mathbb{R} \rightarrow \mathbb{R}$, and a suitable "continuity" condition or "coupling condition" at the interface $x=0$, namely

(i) $f, C_{L}, C_{R} \in C^{1}(\mathbb{R})$,

(ii) $F$ and $f$ are differentiable with respect to $\xi$ and $\zeta$,

(iii) $\xi$ and $\zeta$ are fractionally differentiable with respect to $x$ and $t$,

(iv) $\mu(\tau) t^{\alpha(\tau)} F_{\xi}(\xi)+\beta(\tau) \xi^{\beta(\tau)-1} \neq 0$ for $x<0$ and $\eta(\tau) t^{\gamma(\tau)} F_{\zeta}(\zeta)+\lambda(\tau) \xi^{\lambda(\tau)-1} \neq 0$ for $x>0$, are satisfied.

Then there exists a unique solution $u:(x, t) \in \mathbb{R} \times \mathbb{R}_{+} \rightarrow u(x, t) \in \mathbb{R}$ of (3.3) for $0<$ $\alpha(\tau), \beta(\tau), \gamma(\tau), \lambda(\tau) \leq 1$.

Proof (i) The existence of the solution for (3.3). We implement the method of arbitraryorder fractional characteristics. To construct the continuous solutions, we consider the total differential "du" given by

$$
\begin{aligned}
& \begin{cases}d u=\frac{1}{\Gamma(1+\alpha(\tau))} \frac{\partial^{\alpha(\tau)} u}{\partial \alpha^{\alpha(\tau)}}(d t)^{\alpha(\tau)}+\frac{1}{\Gamma(1+\beta(\tau))} \frac{\partial^{\beta(\tau)} u}{\partial x^{\beta(\tau)}}(d x)^{\beta(\tau)}, & x<0, \\
d u=\frac{1}{\Gamma(1+\gamma(\tau))} \frac{\partial^{\gamma(\tau)} u}{\partial \gamma^{\prime(\tau)}}(d t)^{\gamma(\tau)}+\frac{1}{\Gamma(1+\lambda(\tau))} \frac{\partial^{\lambda(\tau)} u}{\partial x^{\lambda(\tau)}}(d x)^{\lambda(\tau)}, & x>0,\end{cases} \\
& 0<\alpha(\tau), \beta(\tau), \gamma(\tau), \lambda(\tau) \leq 1,
\end{aligned}
$$

so that the points $(x, t)$ are assumed to lie on a curve $\Upsilon$ (upsilon). Then (3.4) can be written as:

$$
\begin{cases}\frac{d u}{(d t)^{\alpha(\tau)}}=\frac{\partial^{\alpha(\tau)} u}{\partial t^{\alpha(\tau)}}+\frac{\Gamma(1+\alpha(\tau))}{\Gamma(1+\beta(\tau))} \frac{\partial^{\beta(\tau)} u}{\partial x^{\beta(\tau)}} \frac{(d x)^{\beta(\tau)}}{(d t)^{\alpha(\tau)},}, & x<0, \\ \frac{d u}{(d t)^{\gamma(\tau)}}=\frac{\partial^{\gamma(\tau)} u}{\partial t^{\gamma(\tau)}}+\frac{\Gamma(1+\gamma(\tau))}{\Gamma(1+\lambda(\tau))} \frac{\partial^{\lambda(\tau)}}{\partial x^{\lambda(\tau)}}\left(\frac{(d x)^{\lambda(\tau)}}{(d t)^{\gamma(\tau)}},\right. & x>0 .\end{cases}
$$

Comparing (3.3) with (3.5), we deduce the following FODEs:

$$
\begin{cases}\frac{d u}{(d t)^{\alpha(\tau)}}=0, & x<0, \\ \frac{d u}{(d t)^{\gamma(\tau)}}=0, & x>0,\end{cases}
$$




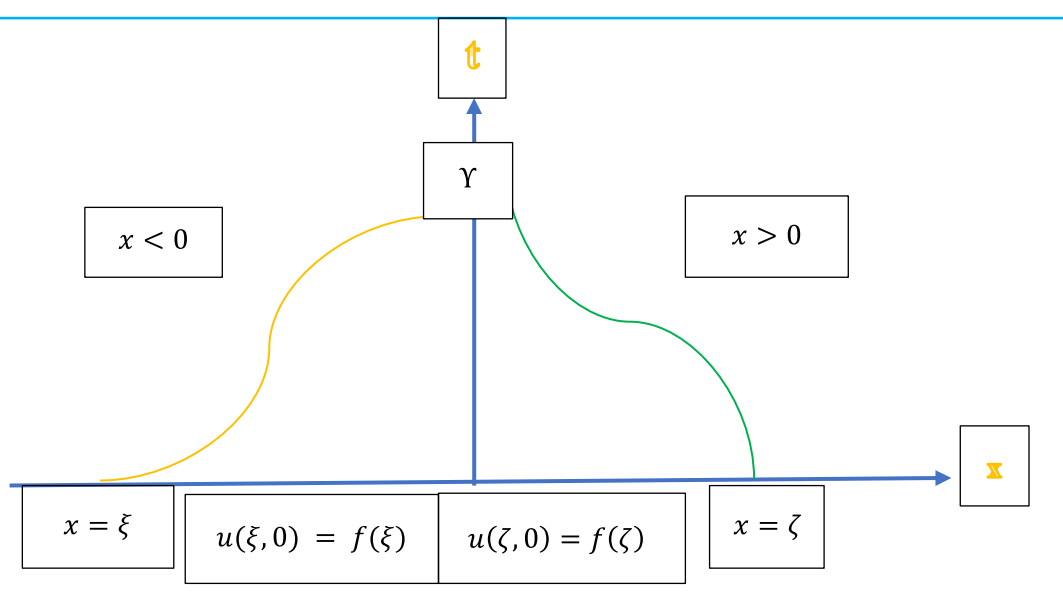

Figure 1 Characteristics curves in the $(x, t)$-plane

and

$$
\begin{cases}\frac{(d x)^{\beta(\tau)}}{(d t)^{\alpha(\tau)}}=\frac{\Gamma(1+\beta(\tau))}{\Gamma(1+\alpha(\tau))} C_{L}(u), & x<0, \\ \frac{(d x)^{\lambda(\tau)}}{(d t)^{\gamma(\tau)}}=\frac{\Gamma(1+\lambda(\tau))}{\Gamma(1+\gamma(\tau))} C_{R}(u), & x>0 .\end{cases}
$$

The solutions of (3.7) are called the fractional characteristic curves of equation (3.3). Thus the solution of (3.3) is reduced to finding the solution of a quadruple of simultaneous ordinary differential equations (3.6) and (3.7).

From (3.6), $u$ is constant along each characteristic curve and each $C_{L}(u)$ or $C_{R}(u)$ remains constant on $\Upsilon$. Hence (3.7) gives the characteristic curves of (3.3), which form a family of curves in the $(x, t)$-plane. It means that if the $\Upsilon$ family of curves can be obtained, then the general solution of (3.3) is obtained. If we assume that the initial condition on the characteristic curve $\Upsilon$ is given by $\xi$ if $x<0$ and $\zeta$ if $x>0$, then $\Upsilon$ intersects $t=0$ when $x<0$ at $x=\xi$, infering $u(\xi, 0)=f(\xi)$ whilst if $x>0$ at $x=\zeta$ then $u(\zeta, 0)=f(\zeta)$ on the entire curve of $\Upsilon$ as shown in Fig. 1.

Thus, the family of the fractional characteristic curves of $\Upsilon$ is the solution of (3.7). Then

$$
\left\{\begin{array}{lll}
\int d u=0, & x<0, & u(\xi, 0)=f(\xi) \\
\int d u=0, & x>0, & u(\zeta, 0)=f(\zeta)
\end{array}\right.
$$

and

$$
\begin{cases}\frac{(d x)^{\beta(\tau)}}{(d t)^{\alpha(\tau)}}=\mu(\tau) C_{L}(u), & x<0, \\ \frac{(d x)^{\lambda(\tau)}}{(d t)^{\gamma(\tau)}}=\eta(\tau) C_{R}(u), & x>0,\end{cases}
$$

where $\mu(\tau)=\frac{\Gamma(1+\beta(\tau))}{\Gamma(1+\alpha(\tau))}$ and $\eta(\tau)=\frac{\Gamma(1+\lambda(\tau))}{\Gamma(1+\gamma(\tau))}$. Equations (3.8) and (3.9) indicate a quadruple of FODEs on $\Upsilon$. However, equation (3.9) cannot be solved because $C_{L}$ and $C_{R}$ are functions of $u$, but (3.8) can easily be solved to obtain $u=$ constant, so $u(\xi, 0)=f(\xi)$ for $x<0$ and 
$u(\zeta, 0)=f(\zeta)$ for $x>0$ on the entire curves of $\Upsilon$. Hence (3.8) leads to

$$
\begin{cases}u(x, t)=f(\xi), & \text { for } x<0 \\ u(x, t)=f(\zeta), & \text { for } x>0\end{cases}
$$

and the integration of (3.9) gives

$$
\left\{\begin{array}{lll}
\int(d x)^{\beta(\tau)}=\mu(\tau) F(\xi) \int(d t)^{\alpha(\tau)}, & x(0)=\xi, & x<0, \\
\int(d x)^{\lambda(\tau)}=\eta(\tau) F(\zeta) \int(d t)^{\gamma(\tau)}, & x(0)=\zeta, & x>0,
\end{array}\right.
$$

where

$$
\begin{cases}F(\xi)=C_{L}(f(\xi)), & x<0 \\ F(\zeta)=C_{R}(f(\zeta)), & x>0\end{cases}
$$

Using formula (2.13), from (3.11) we obtain the following result:

$$
\begin{cases}x^{\beta(\tau)}=\mu(\tau) t^{\alpha(\tau)} F(\xi)+\xi^{\beta(\tau)}, & x<0, \\ x^{\lambda(\tau)}=\eta(\tau) t^{\gamma(\tau)} F(\zeta)+\zeta^{\lambda(\tau)}, & x>0,\end{cases}
$$

which are the characteristic curves of (3.3) (they are straight lines when $\alpha=\beta=\gamma=\lambda=1$ ). The values of $u$ on the curves (3.13) are constant, and it depends on $\xi$ for $x<0$ and $\zeta$ for $x>0$. Therefore (3.10) and (3.13) are the solution of the initial-value problem (3.3), which can be presented in parametric form as

$$
\begin{cases}u(x, t)=f(\xi), & x<0, \\ u(x, t)=f(\zeta), & x>0, \\ x^{\beta(\tau)}=\mu(\tau) t^{\alpha(\tau)} F(\xi)+\xi^{\beta(\tau)}, & x<0, \\ x^{\lambda(\tau)}=\eta(\tau) t^{\gamma(\tau)} F(\zeta)+\zeta^{\lambda(\tau)}, & x>0,\end{cases}
$$

where

$$
\begin{cases}F(\xi)=C_{L}(f(\xi)), & x<0 \\ F(\zeta)=C_{R}(f(\zeta)), & x>0\end{cases}
$$

(ii) Demonstrating (3.14) is a solution of (3.3). We show that (3.14) satisfies (3.3). Differentiating the first and second equations of (3.14) with respect to $x$ with an order of $\beta(\tau)$ and $\gamma(\tau)$, and with respect to $t$ with an order of $\alpha(\tau)$ and $\lambda(\tau)$ yields

$$
\begin{cases}D_{x}^{\beta(\tau)} u(x, t)=D_{x}^{\beta(\tau)} f(\xi), & x<0, \\ D_{t}^{\alpha(\tau)} u(x, t)=D_{t}^{\alpha(\tau)} f(\xi), & x<0, \\ D_{x}^{\lambda(\tau)} u(x, t)=D_{x}^{\lambda(\tau)} f(\xi), & x>0, \\ D_{t}^{\gamma(\tau)} u(x, t)=D_{t}^{\gamma(\tau)} f(\xi), & x>0,\end{cases}
$$


leading to

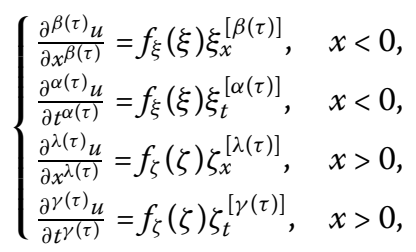

where $f_{\xi}(\xi)=D_{\xi} f(\xi), \xi_{x}^{[\beta(\tau)]}=D_{x}^{\beta(\tau)} \xi$ and $\xi_{t}^{[\alpha(\tau)]}=D_{t}^{\alpha(\tau)} \xi$ (similar for $\zeta$ ). Also, we differentiate the third and fourth equation of (3.14) with respect to $x$ with an order of $\beta(\tau)$ and $\gamma(\tau)$, and with respect to $t$ with an order of $\alpha(\tau)$ and $\lambda(\tau)$ to get

$$
\begin{cases}D_{x}^{\beta(\tau)} x^{\beta(\tau)}=D_{x}^{\beta(\tau)}\left[\mu(\tau) t^{\alpha(\tau)} F(\xi)+\xi^{\beta(\tau)}\right], & x<0, \\ D_{t}^{\alpha(\tau)} x^{\beta(\tau)}=D_{t}^{\alpha(\tau)}\left[\mu(\tau) t^{\alpha(\tau)} F(\xi)+\xi^{\beta(\tau)}\right], & x<0, \\ D_{x}^{\lambda(\tau)} x^{\lambda(\tau)}=D_{x}^{\lambda(\tau)}\left[\eta(\tau) t^{\gamma(\tau)} F(\zeta)+\zeta^{\lambda(\tau)}\right], & x>0, \\ D_{t}^{\gamma(\tau)} x^{\lambda(\tau)}=D_{t}^{\gamma(\tau)}\left[\eta(\tau) t^{\gamma(\tau)} F(\zeta)+\zeta^{\lambda(\tau)}\right], & x>0,\end{cases}
$$

where using formula (2.10) leads to

$$
\begin{cases}\Gamma(1+\beta(\tau))=\left[\mu(\tau) t^{\alpha(\tau)} F_{\xi}(\xi)+\beta \xi^{\beta(\tau)-1}\right] \xi_{x}^{[\beta(\tau)]}, & x<0, \\ 0=\left[\mu(\tau) t^{\alpha(\tau)} F_{\xi}(\xi)+\beta(\tau) \xi^{\beta(\tau)-1}\right] \xi_{t}^{[\alpha(\tau)]}+\Gamma(1+\beta(\tau)) F(\xi), & x<0, \\ \Gamma(1+\lambda(\tau))=\left[\eta(\tau) t^{\gamma(\tau)} F_{\zeta}(\zeta)+\lambda \zeta^{\lambda(\tau)-1}\right] \zeta_{x}^{[\lambda(\tau)]}, & x>0, \\ 0=\left[\eta(\tau) t^{\gamma(\tau)} F_{\zeta}(\zeta)+\lambda(\tau) \zeta^{\lambda(\tau)-1}\right] \zeta_{t}^{[\gamma(\tau)]}+\Gamma(1+\lambda(\tau)) F(\zeta), & x>0 .\end{cases}
$$

Obtaining $\xi_{x}^{[\beta(\tau)]}, \xi_{t}^{[\alpha(\tau)]}, \zeta_{x}^{[\lambda(\tau)]}$ and $\zeta_{t}^{[\gamma(\tau)]}$ from (3.18), and substituting into (3.16), we have

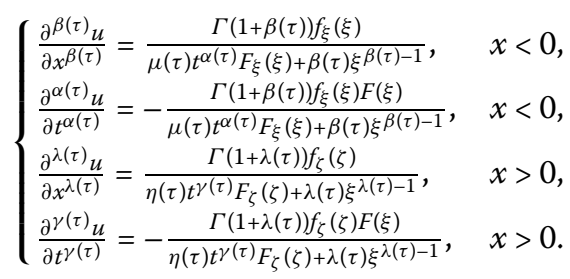

Substituting (3.19) into (3.3), we have

$$
\begin{cases}-\frac{\Gamma(1+\beta(\tau)) f_{\xi}(\xi) F(\xi)}{\mu(\tau) t^{\alpha(\tau)} F_{\xi}(\xi)+\beta(\tau) \xi^{\beta(\tau)-1}}+C_{L}(u(x, t)) \frac{\Gamma(1+\beta(\tau)) f_{\xi}(\xi)}{\mu(\tau) t^{\alpha(\tau)} F_{\xi}(\xi)+\beta(\tau) \xi^{\beta(\tau)-1}}=0, & x<0, t>0, \\ -\frac{\Gamma(1+\lambda(\tau)) f_{\zeta}(\zeta) F(\xi)}{\eta(\tau) t^{\gamma(\tau)} F_{\zeta}(\zeta)+\lambda(\tau) \xi^{\lambda(\tau)-1}}+C_{R}(u(x, t)) \frac{\Gamma(1+\lambda(\tau)) f_{\zeta}(\xi)}{\eta(\tau) t^{\prime}(\tau) F_{\zeta}(\zeta)+\lambda(\tau) \xi^{\lambda(\tau)-1}}=0, & x>0, t>0,\end{cases}
$$

since $F(\xi)=C_{L}(f(\xi))$ for $x<0$ and $F(\zeta)=C_{R}(f(\zeta))$ for $x>0$, equation (3.3) is satisfied provided

$$
\begin{cases}\mu(\tau) t^{\alpha(\tau)} F_{\xi}(\xi)+\beta(\tau) \xi^{\beta(\tau)-1} \neq 0, & x<0 \\ \eta(\tau) t^{\gamma(\tau)} F_{\zeta}(\zeta)+\lambda(\tau) \xi^{\lambda(\tau)-1} \neq 0, & x>0 .\end{cases}
$$

The solution (3.14) also satisfies the initial condition at $t=0$, since $x=\xi$ for $x<0$ and $x=\zeta$ for $x>0$. 
(iii) Showing the uniqueness. Assume that $u(x, t)$ and $v(x, t)$ are two solutions of (3.3). Then they should satisfy (3.14), that is,

$$
\begin{cases}u(x, t)=f(\xi), & x<0, \\ u(x, t)=f(\zeta), & x>0, \\ x^{\beta(\tau)}=\mu(\tau) t^{\alpha(\tau)} F(\xi)+\xi^{\beta(\tau)}, & x<0, \\ x^{\lambda(\tau)}=\eta(\tau) t^{\gamma(\tau)} F(\zeta)+\xi^{\lambda(\tau)}, & x>0,\end{cases}
$$

and

$$
\begin{cases}v(x, t)=f(\xi), & x<0, \\ \nu(x, t)=f(\zeta), & x>0, \\ x^{\beta(\tau)}=\mu(\tau) t^{\alpha(\tau)} F(\xi)+\xi^{\beta(\tau)}, & x<0, \\ x^{\lambda(\tau)}=\eta(\tau) t^{\gamma(\tau)} F(\zeta)+\zeta^{\lambda(\tau)}, & x>0 .\end{cases}
$$

Therefore from (3.20) and (3.21), we obtain

$$
\begin{cases}u(x, t)=f(\xi)=v(x, t), & x<0, \\ u(x, t)=f(\zeta)=v(x, t), & x>0 .\end{cases}
$$

Alternatively,

$$
\begin{cases}x^{\beta(\tau)}=\mu(\tau) t^{\alpha(\tau)} F(\xi)+\xi^{\beta(\tau)}, & x<0, \\ x^{\lambda(\tau)}=\eta(\tau) t^{\gamma(\tau)} F(\zeta)+\zeta^{\lambda(\tau)}, & x>0 .\end{cases}
$$

Hence (3.22) implies that

$$
\begin{cases}\mathrm{u}(x, t)=u(\xi, 0)=f(\xi)=v(x, t), & x<0, \\ \mathrm{u}(x, t)=u(\zeta, 0)=f(\zeta)=v(x, t), & x>0,\end{cases}
$$

so uniqueness is proved.

\section{Application}

Consider the arbitrary-order fractional interface coupling of two nonlinear hyperbolic equations

$$
\begin{aligned}
& \begin{cases}\partial_{t}^{\alpha(\tau)} u(x, t)+C_{L}(u(x, t)) \partial_{x}^{\beta(\tau)} u(x, t)=0, & x<0, t>0, \\
\partial_{t}^{\gamma(\tau)} u(x, t)+C_{R}(u(x, t)) \partial_{x}^{\lambda(\tau)} u(x, t)=0, & x>0, t>0,\end{cases} \\
& 0<\alpha(\tau), \beta(\tau), \gamma(\tau), \lambda(\tau) \leq 1, \\
& u(x, 0)=f(x), \quad x \in \mathbb{R},
\end{aligned}
$$

with an initial condition $u(x, 0): \mathbb{R} \rightarrow \mathbb{R}$ and also a suitable "continuity" condition or "coupling condition" at the interface $x=0$. 
To make this coupling condition more explicit, we begin by considering the simplest possible case where $C_{L}(u)$ and $C_{R}(u)$ are nonzero constants. For the classic case, where $\alpha(\tau)=\beta(\tau)=\gamma(\tau)=\lambda(\tau)=1 \mathrm{E}$. Godlewski [49] has given the following results; the distinguished cases depend on the directions of the characteristic lines:

(1) $C_{L}(u)>0, C_{R}(u)>0$ or $C_{L}(u)<0, C_{R}(u)<0$ : we can impose the continuity of $u$ at $x=0$;

(2) $C_{L}(u)>0, C_{R}(u)<0$ : no continuity condition is required at $x=0$;

(3) $C_{L}(u)<0, C_{R}(u)>0$ : we need to specify $u(0, t)$ at $x=0$, otherwise the solution $u$ is not defined in the fan $C_{L} t<x<C_{R} t$.

Let us examine the above result by the following example.

Example 1 We obtain the analytical solution for interface coupling space-time fractional arbitrary-order equations of fluid mechanics that are dealing with two different complex systems of equations on each side of the interface, which in this case are $C_{L}(u)=$ constant $=$ $k$ and $C_{R}(u)=$ constant $=h$. The system is

$$
\begin{aligned}
& \begin{cases}\frac{\partial^{\alpha(\tau)} u}{\partial t^{\alpha(\tau)}}+k \frac{\partial^{\beta(\tau)} u}{\partial x^{\beta(\tau)}}=0, & x<0, \\
\frac{\partial^{\gamma(\tau)} u}{\partial t^{\gamma(\tau)}}+h \frac{\partial^{\lambda(\tau)} u}{\partial x^{\lambda(\tau)}}=0, & x>0,\end{cases} \\
& 0<\alpha(\tau), \beta(\tau), \gamma(\tau), \lambda(\tau) \leq 1, \\
& u(x, 0)=f(x)=\sin 2 x+5 \cos x .
\end{aligned}
$$

Solution According to Theorem 1, we can write the following FPDEs:

$$
\left\{\begin{array}{lrl}
\frac{d u}{(d t)^{\alpha(\tau)}}=0, & u(\xi, 0)=f(\xi), & x<0, \\
\frac{d u}{(d t)^{\gamma(\tau)}}=0, & u(\zeta, 0)=f(\zeta), & x>0, \\
\frac{d d x)^{\beta(\tau)}}{(d t)^{\alpha(\tau)}}=\mu(\tau) k, & x(0)=\xi, & x<0, \\
\frac{d d x)^{\lambda(\tau)}}{(d t)^{\gamma(\tau)}}=\eta(\tau) h, & x(0)=\zeta, & x>0,
\end{array}\right.
$$

where $\mu(\tau)=\frac{\Gamma(1+\beta(\tau))}{\Gamma(1+\alpha(\tau))}$ and $\eta(\tau)=\frac{\Gamma(1+\lambda(\tau))}{\Gamma(1+\gamma(\tau))}$. Integrating (4.4), we have

$$
\left\{\begin{array}{lrr}
\int d u=0, \quad u(\xi, 0)=f(\xi), & & x<0, \\
\int d u=0, \quad u(\zeta, 0)=f(\zeta), & & x>0, \\
\int(d x)^{\beta(\tau)}=\mu(\tau) k \int(d t)^{\alpha(\tau)}, & x(0)=\xi, & x<0, \\
\int(d x)^{\lambda(\tau)}=\eta(\tau) h \int(d t)^{\gamma(\tau)}, & x(0)=\zeta, & x>0,
\end{array}\right.
$$

then, implementing formula (2.13), we obtain the parametric solution

$$
\begin{cases}u(x, t)=f(\xi), & x<0, \\ u(x, t)=f(\zeta), & x>0, \\ x^{\beta(\tau)}=\mu(\tau) k t^{\alpha(\tau)}+\xi^{\beta(\tau)}, & x<0, \\ x^{\lambda(\tau)}=\eta(\tau) h t^{\gamma(\tau)}+\xi^{\lambda(\tau)}, & x>0,\end{cases}
$$


since

$$
\begin{cases}u(x, t)=f(\xi), & x<0 \\ u(x, t)=f(\zeta), & x>0 \\ \xi=\left(x^{\beta(\tau)}-\mu(\tau) k t^{\alpha(\tau)}\right)^{1 / \beta(\tau)}, & x<0 \\ \zeta=\left(x^{\lambda(\tau)}-\eta(\tau) h t^{\gamma(\tau)}\right)^{1 / \lambda(\tau)}, & x>0\end{cases}
$$

and therefore the solution can be written as

$$
\begin{cases}u(x, t)=f(\xi)=f\left(\left(x^{\beta(\tau)}-\mu(\tau) k t^{\alpha(\tau)}\right)^{1 / \beta(\tau)}\right), & x<0, \\ u(x, t)=f(\zeta)=f\left(\left(x^{\lambda(\tau)}-\eta(\tau) h t^{\gamma(\tau)}\right)^{1 / \lambda(\tau)}\right), & x>0 .\end{cases}
$$

The general solution for Example 1, assuming the initial condition $u(x, 0)=f(x)=\sin 2 x+$ $5 \cos x$, is

$$
\left\{\begin{array}{l}
u(x, t)=\sin 2\left(x^{\beta(\tau)}-\mu(\tau) k t^{\alpha(\tau)}\right)^{1 / \beta(\tau)}+5 \cos \left(x^{\beta(\tau)}-\mu(\tau) k t^{\alpha(\tau)}\right)^{1 / \beta(\tau)} \\
\quad x<0 \\
u(x, t)=\sin 2\left(x^{\lambda(\tau)}-\eta(\tau) h t^{\gamma(\tau)}\right)^{1 / \lambda(\tau)}+5 \cos \left(x^{\lambda(\tau)}-\eta(\tau) h t^{\gamma(\tau)}\right)^{1 / \lambda(\tau)} \\
\quad x>0
\end{array}\right.
$$

Benchmark 1 (For Example 1) We make sure that (4.6) is the solution, and satisfies (4.2). Differentiating the first and second equation of (4.6) with respect to $x$ with an order of $\beta(\tau)$ and $\gamma(\tau)$, and with respec to $t$ with an order of $\alpha(\tau)$ and $\lambda(\tau)$ gives

$$
\begin{cases}D_{x}^{\beta(\tau)} u(x, t)=D_{x}^{\beta(\tau)} f(\xi), & x<0 \\ D_{t}^{\alpha(\tau)} u(x, t)=D_{t}^{\alpha(\tau)} f(\xi), & x<0 \\ D_{x}^{\lambda(\tau)} u(x, t)=D_{x}^{\lambda(\tau)} f(\xi), & x>0 \\ D_{t}^{\gamma(\tau)} u(x, t)=D_{t}^{\gamma(\tau)} f(\xi), & x>0\end{cases}
$$

leading to

$$
\begin{cases}\frac{\partial^{\beta(\tau)} u}{\partial x^{\beta(\tau)}}=f_{\xi}(\xi) \xi_{x}^{[\beta(\tau)]}, & x<0 \\ \frac{\partial^{\alpha(\tau)} u}{\partial t^{\alpha(\tau)}}=f_{\xi}(\xi) \xi_{t}^{[\alpha(\tau)]}, & x<0 \\ \frac{\partial^{\lambda(\tau)} u}{\partial x^{\lambda(\tau)}}=f_{\zeta}(\zeta) \zeta_{x}^{[\lambda(\tau)]}, & x>0 \\ \frac{\partial^{\gamma(\tau)} u}{\partial t^{\gamma(\tau)}}=f_{\zeta}(\zeta) \zeta_{t}^{[\gamma(\tau)]}, & x>0\end{cases}
$$

Also, we differentiate the third and fourth equation of (4.6) with respect to $x$ with an order of $\beta(\tau)$ and $\gamma(\tau)$, and with respect to $t$ with an order of $\alpha(\tau)$ and $\lambda(\tau)$ to get

$$
\begin{cases}D_{x}^{\beta(\tau)} x^{\beta(\tau)}=D_{x}^{\beta(\tau)}\left[\mu(\tau) k t^{\alpha(\tau)}+\xi^{\beta(\tau)}\right], & x<0, \\ D_{t}^{\alpha(\tau)} x^{\beta(\tau)}=D_{t}^{\alpha(\tau)}\left[\mu(\tau) k t^{\alpha(\tau)}+\xi^{\beta(\tau)}\right], & x<0, \\ D_{x}^{\lambda(\tau)} x^{\lambda(\tau)}=D_{x}^{\lambda(\tau)}\left[\eta(\tau) h t^{\gamma(\tau)}+\zeta^{\lambda(\tau)}\right], & x>0, \\ D_{t}^{\gamma(\tau)} x^{\lambda(\tau)}=D_{t}^{\gamma(\tau)}\left[\eta(\tau) h t^{\gamma(\tau)}+\zeta^{\lambda(\tau)}\right], & x>0\end{cases}
$$


which, using formula (2.10), leads to

$$
\begin{cases}\Gamma(1+\beta(\tau))=\beta(\tau) \xi^{\beta(\tau)-1} \xi_{x}^{[\beta(\tau)]}, & x<0, \\ 0=\Gamma(1+\beta(\tau)) k+\beta(\tau) \xi^{\beta(\tau)-1} \xi_{t}^{[\alpha(\tau)]}, & x<0, \\ \Gamma(1+\lambda(\tau))=\lambda(\tau) \zeta^{\lambda(\tau)-1} \zeta_{x}^{[\lambda(\tau)]}, & x>0, \\ 0=\Gamma(1+\lambda(\tau)) h+\lambda(\tau) \zeta^{\lambda(\tau)-1} \zeta_{t}^{[\gamma(\tau)]}, & x>0 .\end{cases}
$$

Obtaining $\xi_{x}^{[\beta(\tau)]}, \xi_{t}^{[\alpha(\tau)]}, \zeta_{x}^{[\lambda(\tau)]}$ and $\zeta_{t}^{[\gamma(\tau)]}$ from (4.13),

$$
\begin{cases}\xi_{x}^{[\beta(\tau)]}=\frac{\Gamma(1+\beta(\tau))}{\beta(\tau) \xi^{\beta(\tau)-1},} & x<0, \\ \xi_{t}^{[\alpha(\tau)]}=-\frac{\Gamma(1+\beta(\tau)) k}{\beta(\tau) \xi^{\beta(\tau)-1}}, & x<0, \\ \zeta_{x}^{[\lambda(\tau)]}=\frac{\Gamma(1+\lambda(\tau))}{\lambda(\tau) \zeta^{\lambda(\tau)-1},} & x>0, \\ \zeta_{t}^{[\gamma(\tau)]}=-\frac{\Gamma(1+\lambda(\tau)) h}{\lambda(\tau) \zeta^{\lambda(\tau)-1}}, & x>0,\end{cases}
$$

and substituting into (4.11), we have

$$
\begin{cases}\frac{\partial^{\beta(\tau)} u}{\partial x^{\beta(\tau)}}=f_{\xi}(\xi) \frac{\Gamma(1+\beta(\tau))}{\beta(\tau) \xi^{\beta(\tau)-1}}, & x<0 \\ \frac{\partial^{\alpha(\tau)} u}{\partial t^{\alpha(\tau)}}=-f_{\xi}(\xi) \frac{\Gamma(1+\beta(\tau)) k}{\beta(\tau) \xi^{\beta(\tau)-1}}, & x<0 \\ \frac{\partial^{\lambda(\tau) u}}{\partial x^{\lambda(\tau)}}=f_{\zeta}(\zeta) \frac{\Gamma(1+\lambda(\tau))}{\lambda(\tau) \zeta^{\lambda(\tau)-1}}, & x>0 \\ \frac{\partial^{\gamma(\tau) u}}{\partial t^{\gamma(\tau)}}=-f_{\zeta}(\zeta) \frac{\Gamma(1+\lambda(\tau)) h}{\lambda(\tau) \zeta^{\lambda(\tau)-1}}, & x>0 .\end{cases}
$$

Substituting (4.15) into (4.2), we have

$$
\begin{cases}-f_{\xi}(\xi) \frac{\Gamma(1+\beta(\tau)) k}{\beta(\tau) \xi^{\beta(\tau)-1}}+k f_{\xi}(\xi) \frac{\Gamma(1+\beta(\tau))}{\beta(\tau) \xi^{\beta(\tau)-1}}=0, & x<0, \\ -f_{\zeta}(\zeta) \frac{\Gamma(1+\lambda(\tau)) h}{\lambda(\tau) \zeta^{\lambda(\tau)-1}}+h f_{\zeta}(\zeta) \frac{\Gamma(1+\lambda(\tau))}{\lambda(\tau) \zeta^{\lambda(\tau)-1}}=0, & x>0,\end{cases}
$$

therefore (4.6) is the solution of (4.2). The answer (4.6) also satisfies the initial condition at $t=0$, since $x=\xi$ for $x<0$ and $x=\zeta$ for $x>0$.

Remark 2 We consider the classic case of Example 1, where $\alpha(\tau)=\beta(\tau)=\gamma(\tau)=\lambda(\tau)=1$ :

$$
\begin{cases}\frac{\partial u}{\partial t}+k \frac{\partial u}{\partial x}=0, & x<0 \\ \frac{\partial u}{\partial t}+h \frac{\partial u}{\partial x}=0, & x>0\end{cases}
$$

with the initial condition

$$
u(x, 0)=f(x)=\sin 2 x+5 \cos x
$$

The solution $u$ is

$$
\begin{cases}u(x, t)=\sin 2(x-k t)+5 \cos (x-k t), & x<0, \\ u(x, t)=\sin 2(x-h t)+5 \cos (x-h t), & x>0 .\end{cases}
$$


The graphs of the solution $u$ for different values of $k$ and $h$ are given in Fig. 2. Each graph of the solution $u$ for various values of $h$ and $k$ has only real part that is shown in segments (a), in which time is continuous, and in segments (b) when time is $t=0,1,2,3,4$, and 5.

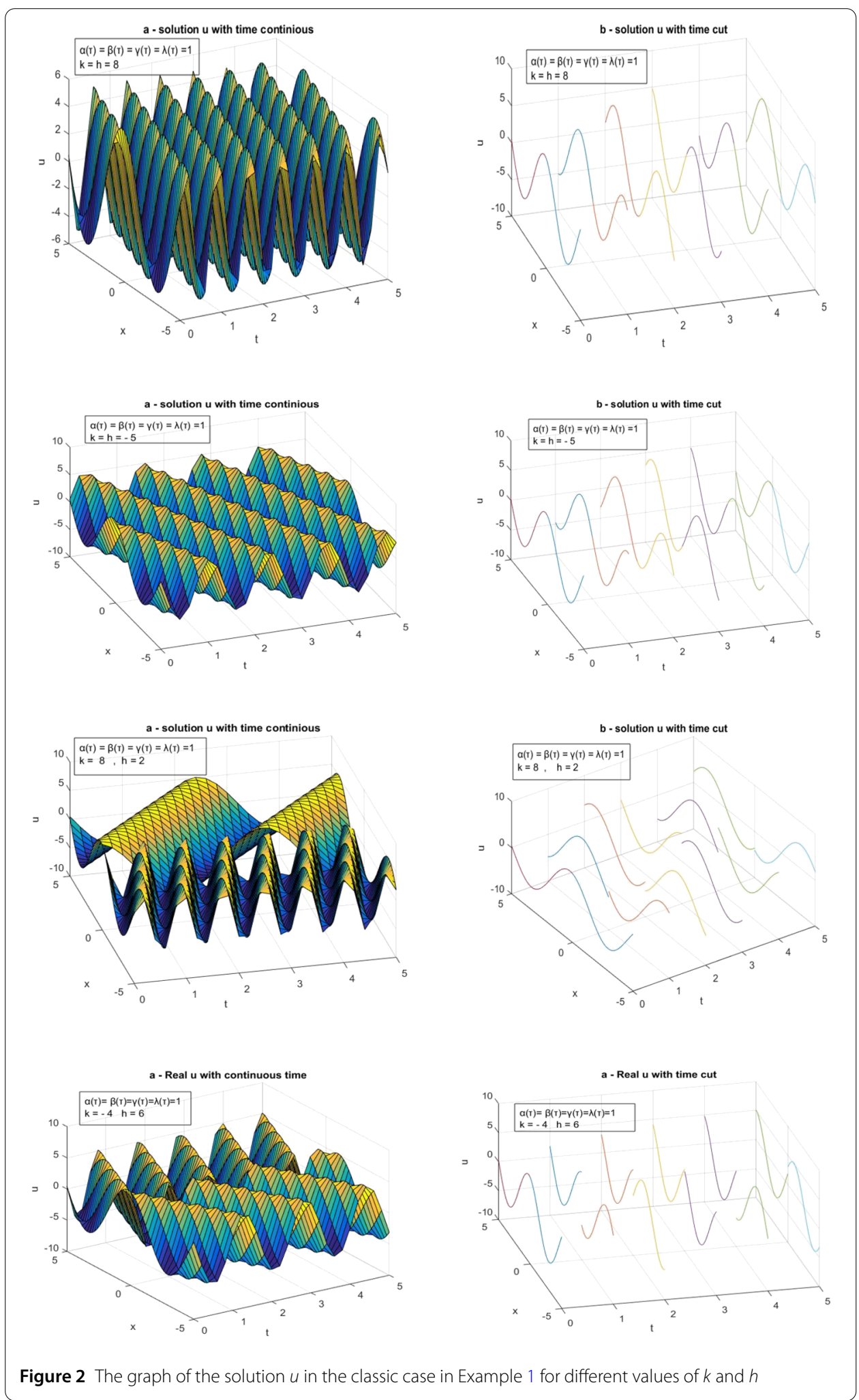


Also, we consider Example 1 with other values for $\alpha(\tau), \beta(\tau), \gamma(\tau)$, and $\lambda(\tau)$ and the graphs of $u$ are given in Fig. 3. In these cases, the solution $u$ has real and imaginary parts.

Remark 3 We can conclude from the analysis of results in Example 1 that the solution $u$ at the interface $x=0$ is continuous when $C_{L}(u)=k=h=C_{R}(u)$ both in classic and fractional cases, which matches the result by E. Godlewski. But when $C_{L}(u)=k \neq h=C_{R}(u)$, the continuity does not hold; therefore, it does not match with the result by E. Godlewski.

Example 2 Here we investigating the solution of a more complicated form of the interface coupling of the space-time fractional arbitrary-order, which is given by

$$
\begin{aligned}
& \begin{cases}e^{t^{\alpha(\tau)}} \frac{\partial^{\alpha(\tau)} u}{\partial^{\alpha(\tau)}}+e^{x^{\beta(\tau)}} \frac{\partial^{\beta(\tau)} u}{\partial^{\beta(\tau)}}=0, & x<0, \\
e^{\gamma^{\gamma(\tau)}} \frac{\partial^{\gamma(\tau)} u}{\partial t^{\gamma(\tau)}}+e^{\lambda^{\lambda(\tau)}} \frac{\partial^{\lambda(\tau)} u}{\partial x^{\lambda(\tau)}}=0, & x>0,\end{cases} \\
& 0<\alpha(\tau), \beta(\tau), \gamma(\tau), \lambda(\tau) \leq 1, \\
& u(x, 0)=f(x)=e^{2 x}+\cos 2 x .
\end{aligned}
$$

Solution According to Theorem 1, for $x<0$, we have

$$
\left\{\begin{array}{l}
\frac{d u}{(d t)^{\alpha(\tau)}}=0, \\
u(x, 0)=f(\xi), \\
\frac{(d x)^{\beta(\tau)}}{(d t)^{\alpha(\tau)}}=\frac{\Gamma(1+\beta(\tau))}{\Gamma(1+\alpha(\tau))} e^{x^{\beta(\tau)}} \\
x(0)=\xi
\end{array}\right.
$$

and for $x>0$, we have

$$
\left\{\begin{array}{l}
\frac{d u}{(d t)^{\gamma(\tau)}}=0, \\
u(x, 0)=f(\zeta), \\
\frac{(d x)^{\lambda(\tau)}}{(d t)^{\gamma(\tau)}}=\frac{\Gamma(1+\lambda(\tau))}{\Gamma(1+\gamma(\tau))} \frac{e^{\lambda^{(\tau)}}}{e^{t^{\gamma(\tau)}}} \\
x(0)=\zeta .
\end{array}\right.
$$

Then the integration of (4.18) gives

$$
\left\{\begin{array}{l}
\int d u=0, \\
u(x, 0)=f(\xi), \\
\int e^{-x^{\beta(\tau)}}(d x)^{\beta(\tau)}=\mu(\tau) \int e^{t^{\alpha(\tau)}}(d t)^{\alpha(\tau)}, \\
x(0)=\xi
\end{array}\right.
$$

where $\mu(\tau)=\frac{\Gamma(1+\beta(\tau))}{\Gamma(1+\alpha(\tau))}$. By formula (2.14) the integration of (4.20) for $x>0$ yields

$$
\left\{\begin{array}{l}
u=C_{1}, \\
u(x, 0)=f(\xi), \\
e^{-x^{\beta(\tau)}}=\mu(\tau) e^{-t^{\alpha(\tau)}}+C_{2}, \\
x(0)=\xi
\end{array}\right.
$$




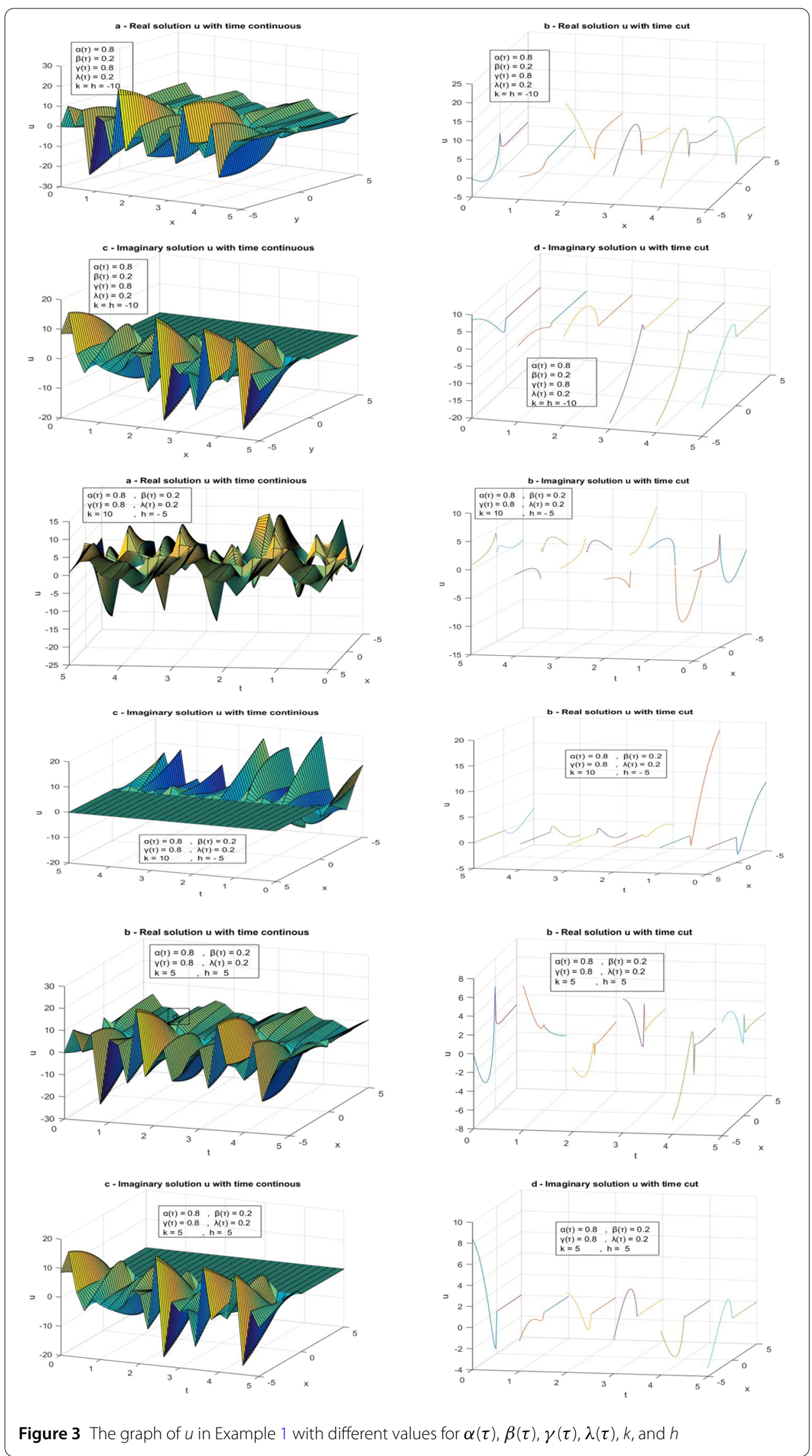


and leads to

$$
\begin{cases}u(x, t)=f(\xi), & x<0, \\ e^{-x^{\beta(\tau)}}=\mu(\tau) e^{-t^{\alpha(\tau)}}+e^{-\xi^{\beta(\tau)}}, & x<0 .\end{cases}
$$

Similar to the above, from (4.19), we obtain

$$
\begin{cases}u(x, t)=f(\zeta), & x>0, \\ e^{-x^{\lambda(\tau)}}=\eta(\tau) e^{-t^{\gamma(\tau)}}+e^{-\zeta^{\lambda(\tau)}}, & x>0,\end{cases}
$$

where $\eta(\tau)=\frac{\Gamma(1+\lambda(\tau))}{\Gamma(1+\gamma(\tau))}$. Therefore, (4.22) and (4.23) give the parametric solution below for (4.17) as

$$
\begin{cases}u(x, t)=f(\xi), & x<0, \\ u(x, t)=f(\zeta), & x>0, \\ e^{-x^{\beta(\tau)}}=\mu(\tau) e^{-t^{\alpha(\tau)}}+e^{-\xi^{\beta(\tau)}}, & x<0, \\ e^{-x^{\lambda(\tau)}}=\eta(\tau) e^{-t^{\gamma(\tau)}}+e^{-\zeta^{\lambda(\tau)}}, & x>0,\end{cases}
$$

and (4.24) can be written in the following form:

$$
\begin{cases}u(x, t)=f(\xi)=f\left(\ln \left(\left(\mu(\tau) e^{-t^{\alpha(\tau)}}-e^{-x^{\beta(\tau)}}\right)^{1 / \beta(\tau)}\right)\right), & x<0, \\ u(x, t)=f(\xi)=f\left(\ln \left(\left(\eta(\tau) e^{-t^{\gamma(\tau)}}-e^{-x^{\lambda(\tau)}}\right)^{1 / \lambda(\tau)}\right)\right), & x>0 .\end{cases}
$$

Considering the initial condition

$$
u(x, 0)=f(x)=e^{2 x}+\cos 2 x
$$

and (4.25), we have

$$
\left\{\begin{array}{rlrl}
u(x, t)= & e^{2 \ln \left(\left(\mu(\tau) e^{-t^{\alpha(\tau)}}-e^{-x^{\beta(\tau)}}\right)^{1 / \beta(\tau)}\right)} & \\
& +\cos \left(5 \ln \left(\left(\mu(\tau) e^{-t^{\alpha(\tau)}}-e^{-x^{\beta(\tau)}}\right)^{1 / \beta(\tau)}\right)\right), & & x<0, \\
u(x, t)= & e^{2 \ln \left(\left(\eta(\tau) e^{-t^{\gamma}(\tau)}-e^{-x^{\lambda(\tau)}}\right)^{1 / \lambda(\tau)}\right)} & \\
& +\cos \left(5 \ln \left(\left(\eta(\tau) e^{-t^{\gamma(\tau)}}-e^{-x^{\lambda(\tau)}}\right)^{1 / \lambda(\tau)}\right)\right), & & x>0,
\end{array}\right.
$$

which leads to

$$
\left\{\begin{array}{rlr}
u(x, t)= & \left(\mu(\tau) e^{-t^{\alpha(\tau)}}-e^{-x^{\beta(\tau)}}\right)^{2 / \beta(\tau)} & \\
& +\cos \left(5 \ln \left(\mu(\tau) e^{-t^{\alpha(\tau)}}-e^{-x^{\beta(\tau)}}\right)^{1 / \beta(\tau)}\right), \quad & x<0, \\
u(x, t)= & \left(\eta(\tau) e^{-t^{\gamma(\tau)}}-e^{-x^{\lambda(\tau)}}\right)^{2 / \lambda(\tau)} & \\
& +\cos \left(5 \ln \left(\eta(\tau) e^{-t^{\gamma(\tau)}}-e^{-x^{\lambda(\tau)}}\right)^{1 / \lambda(\tau)}\right), \quad & x>0 .
\end{array}\right.
$$

Benchmark 2 (For Example 2) We make sure that (4.24) is the solution, and satisfies (4.17). Differentiating the first and second equation of (4.24) with respect to $x$ with an 
order of $\beta(\tau)$ and $\gamma(\tau)$, and with respect to $t$ with an order of $\alpha(\tau)$ and $\lambda(\tau)$ gives

$$
\begin{cases}D_{x}^{\beta(\tau)} u(x, t)=D_{x}^{\beta(\tau)} f(\xi), & x<0 \\ D_{t}^{\alpha(\tau)} u(x, t)=D_{t}^{\alpha(\tau)} f(\xi), & x<0 \\ D_{x}^{\lambda(\tau)} u(x, t)=D_{x}^{\lambda(\tau)} f(\xi), & x>0 \\ D_{t}^{\gamma(\tau)} u(x, t)=D_{t}^{\gamma(\tau)} f(\xi), & x>0\end{cases}
$$

leading to

$$
\begin{cases}\frac{\partial^{\beta(\tau)} u}{\partial x^{\beta(\tau)}}=f_{\xi}(\xi) \xi_{x}^{[\beta(\tau)]}, & x<0, \\ \frac{\partial^{\alpha(\tau)} u}{\partial t^{\alpha(\tau)}}=f_{\xi}(\xi) \xi_{t}^{[\alpha(\tau)]}, & x<0, \\ \frac{\partial^{\lambda(\tau)} u}{\partial x^{\lambda(\tau)}}=f_{\zeta}(\zeta) \zeta_{x}^{[\lambda(\tau)]}, & x>0, \\ \frac{\partial^{\gamma(\tau)} u}{\partial t^{\gamma}(\tau)}=f_{\zeta}(\zeta) \zeta_{t}^{[\gamma(\tau)],}, & x>0 .\end{cases}
$$

Also, we differentiate the third and fourth equation of (4.24) with respect to $x$ with an order of $\beta(\tau)$ and $\gamma(\tau)$, and with respect to $t$ with an order of $\alpha(\tau)$ and $\lambda(\tau)$ to get

$$
\begin{cases}D_{x}^{\beta(\tau)} e^{-x^{\beta(\tau)}}=D_{x}^{\beta(\tau)}\left[\mu(\tau) e^{-t^{\alpha(\tau)}}+e^{-\xi^{\beta(\tau)}},\right], & x<0, \\ D_{t}^{\alpha(\tau)} e^{-x^{\beta(\tau)}}=D_{t}^{\alpha(\tau)}\left[\mu(\tau) e^{-t^{\alpha(\tau)}}+e^{-\xi^{\beta(\tau)}}\right], & x<0, \\ D_{x}^{\lambda(\tau)} e^{-x^{\lambda(\tau)}}=D_{x}^{\lambda(\tau)}\left[\eta(\tau) e^{-t^{\gamma(\tau)}}+e^{-\zeta^{\lambda(\tau)}}\right], & x>0, \\ D_{t}^{\gamma(\tau)} e^{-x^{\lambda(\tau)}}=D_{t}^{\gamma(\tau)}\left[\eta(\tau) e^{-t^{\gamma(\tau)}}+e^{-\zeta^{\lambda(\tau)}}\right], & x>0,\end{cases}
$$

which, using formula (2.10), leads to

$$
\begin{cases}\Gamma(1+\beta(\tau)) e^{-x^{\beta(\tau)}}=\beta(\tau) \xi^{\beta(\tau)-1} e^{-\xi^{\beta(\tau)}} \xi_{x}^{[\beta(\tau)]}, & x<0, \\ 0=\Gamma(1+\beta(\tau)) e^{-t^{\alpha(\tau)}}+\beta(\tau) \xi^{\beta(\tau)-1} e^{-\xi^{\beta(\tau)}} \xi_{t}^{[\alpha(\tau)]}, & x<0, \\ \Gamma(1+\lambda(\tau)) e^{-x^{\lambda(\tau)}}=\lambda(\tau) \zeta^{\lambda(\tau)-1} e^{-\zeta^{\lambda(\tau)}} \zeta_{x}^{[\lambda(\tau)]}, & x>0, \\ 0=\Gamma(1+\lambda(\tau)) e^{-t^{\gamma(\tau)}}+\lambda(\tau) \zeta^{\lambda(\tau)-1} e^{-\zeta^{\lambda(\tau)}} \zeta_{t}^{[\gamma(\tau)]}, & x>0 .\end{cases}
$$

Obtaining $\xi_{x}^{[\beta(\tau)]}, \xi_{t}^{[\alpha(\tau)]}, \zeta_{x}^{[\lambda(\tau)]}$, and $\zeta_{t}^{[\gamma(\tau)]}$ from (4.31), namely

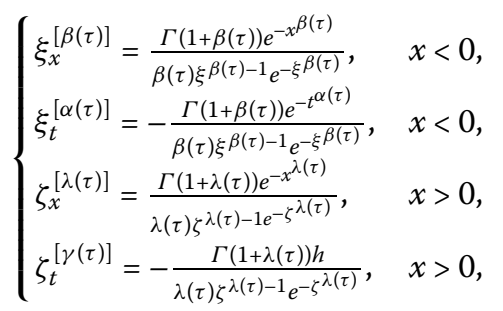

and substituting (4.32) into (4.17), we have

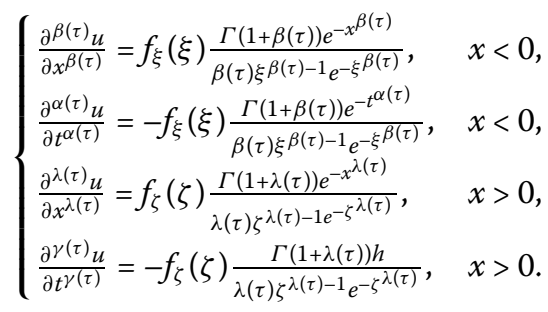


And substituting (4.15) into (4.2), we have

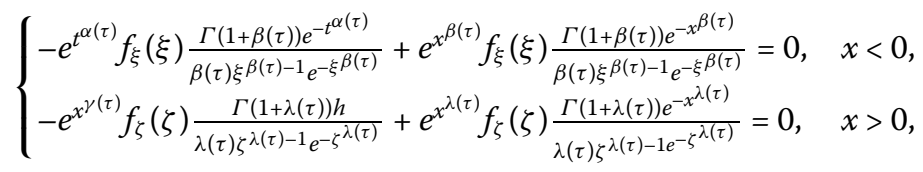

therefore (4.6) is the solution of (4.2). The answer (4.6) also satisfies the initial condition at $t=0$, since $x=\xi$ for $x<0$ and $x=\zeta$ for $x>0$.

Remark 4 Consider the classic case of Example 2 where $\alpha(\tau)=\beta(\tau)=\gamma(\tau)=\lambda(\tau)=1$, therefore the solution is

$$
\begin{cases}u(x, t)=f(\xi)=f\left(\ln \left(e^{-t}-e^{-x}\right)\right), & x<0 \\ u(x, t)=f(\zeta)=f\left(\ln \left(e^{-t}-e^{-x}\right)\right), & x>0 .\end{cases}
$$

With initial condition $u(x, 0)=f(x)=e^{2 x}+\cos 5 x$ the solution can be written as

$$
\begin{cases}u(x, t)=f(\xi)=e^{2 \ln \left(e^{-t}-e^{-x}\right)}+\cos \left(5 \ln \left(e^{-t}-e^{-x}\right)\right), & x<0 \\ u(x, t)=f(\zeta)=e^{2 \ln \left(e^{-t}-e^{-x}\right)}+\cos \left(5 \ln \left(e^{-t}-e^{-x}\right)\right), & x>0\end{cases}
$$

and its graph is given in Fig. 4.

The graphs of the solution $u$ in Example 2 for different values of $\alpha(\tau), \beta(\tau), \gamma(\tau)$, and $\lambda(\tau)$ are given in Fig. 5, where (a) and (c) are the graphs of the real and imaginary part

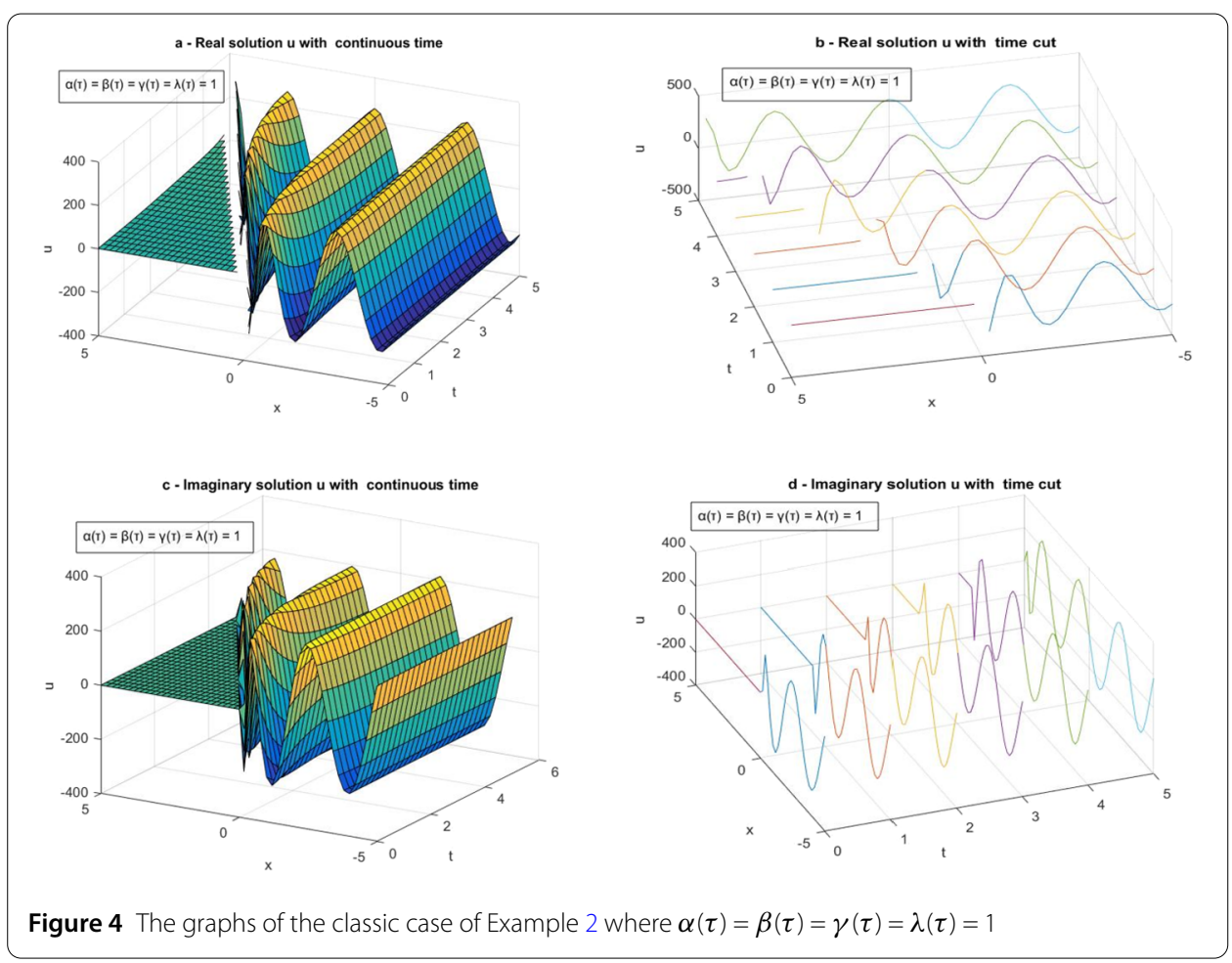




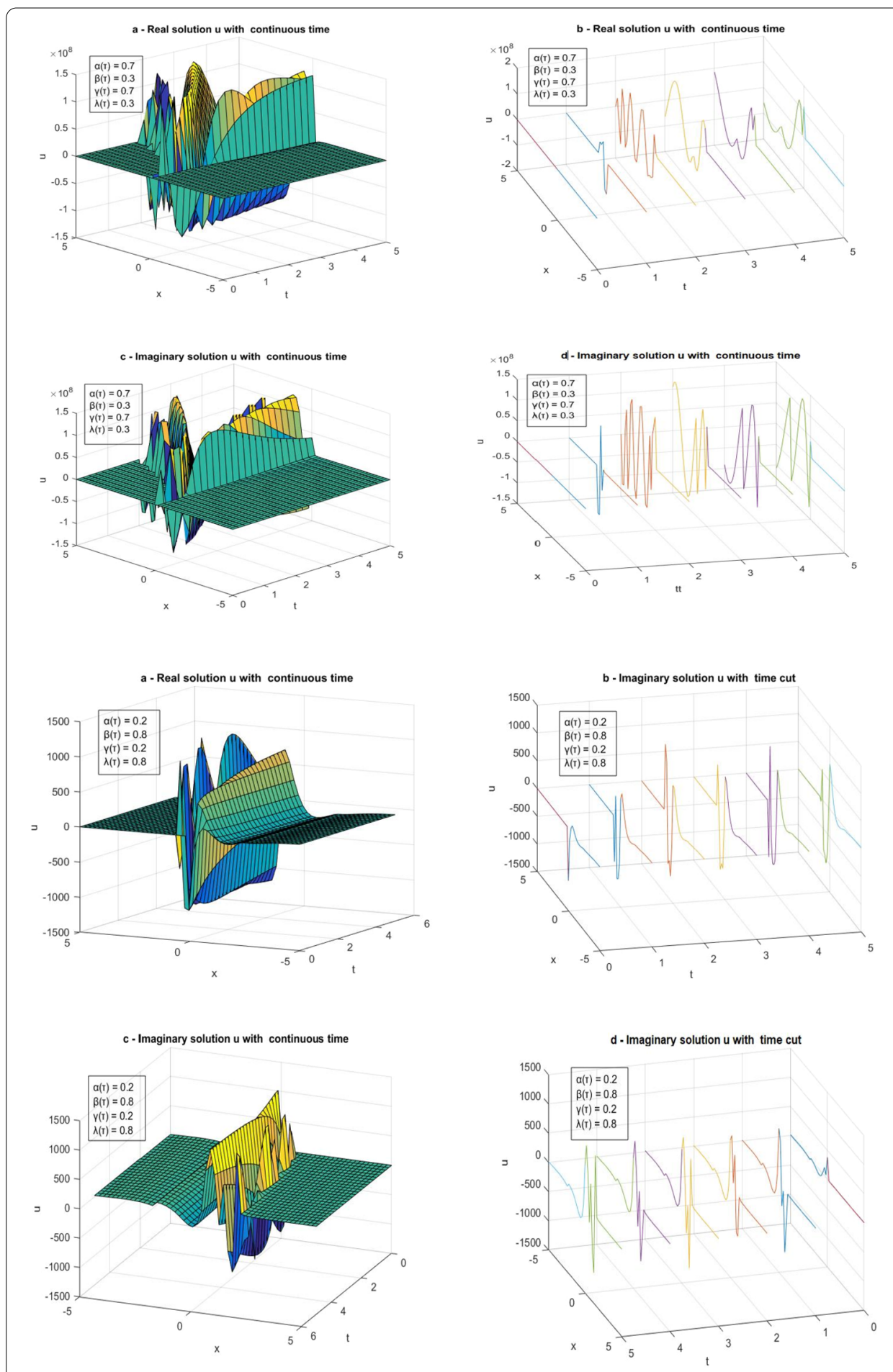

Figure 5 The graphs of the solution $u$ in Example 2 with different values for $\alpha(\tau), \beta(\tau), \gamma(\tau)$, and $\lambda(\tau)$

of the solutions $u$ where time is continuous, and (b) and (d) are the graphs the real and imaginary part of the solution $u$ with the different values for the time at $t=0,1,2,3,4$, and 5 . 
Example 3 Here we find the analytical solution of a couple interface fractional arbitraryorder differential equation

$$
\begin{aligned}
& \begin{cases}\frac{t^{\alpha(\tau)}}{\Gamma(1+\alpha(\tau))} \frac{\partial^{\alpha(\tau)} u}{t^{\alpha(\tau)}}+\frac{3 x^{\beta(\tau)}}{\Gamma(1+\beta(\tau))} \frac{\partial^{\beta(\tau)} u}{\partial x^{\beta(\tau)}}=0, & x<0, \\
\frac{t^{\gamma}(\tau)}{\Gamma(1+\gamma(\tau))} \frac{\partial^{\gamma(\tau)} u}{\partial t^{\gamma}(\tau)}+\frac{5 x^{\lambda(\tau)}}{\Gamma(1+\lambda(\tau))} \frac{\partial^{\lambda(\tau)} u}{\partial x^{\lambda(\tau)}}=0, & x>0,\end{cases} \\
& 0<\alpha(\tau), \beta(\tau), \gamma(\tau), \lambda(\tau) \leq 1, \\
& u(x, 1)=f(x)=\cos x+3 \sin x .
\end{aligned}
$$

Solution Based on the method introduced in Theorem 1, for $x<0$,

$$
\left\{\begin{array}{l}
\frac{d u}{(d t)^{\alpha(\tau)}}=0 \\
u(x, 1)=f(\xi) \\
\frac{\Gamma(1+\alpha(\tau))}{\Gamma(1+\beta(\tau))} \frac{(d x)^{\beta(\tau)}}{(d t)^{\alpha(\tau)}}=3 \frac{\Gamma(1+\alpha(\tau))}{\Gamma(1+\beta(\tau))} \frac{x^{\beta(\tau)}}{t^{\alpha(\tau)}} \\
x(1)=\xi
\end{array}\right.
$$

and for $x>0$,

$$
\left\{\begin{array}{l}
\frac{d u}{(d t)^{\gamma(\tau)}}=0 \\
u(x, 1)=f(\zeta) \\
\frac{\Gamma(1+\gamma(\tau))}{\Gamma(1+\lambda(\tau))} \frac{(d x)^{\lambda(\tau)}}{(d t)^{\gamma(\tau)}}=5 \frac{\Gamma(1+\gamma(\tau))}{\Gamma(1+\lambda(\tau))} \frac{x^{\lambda(\tau)}}{t^{\gamma(\tau)}} \\
x(1)=\zeta .
\end{array}\right.
$$

The integration of (4.37) and (4.38) is given below: for $x<0$, we have

$$
\left\{\begin{array}{l}
\int d u=0, \\
u(x, 1)=f(\xi), \\
\int \frac{1}{x^{\beta(\tau)}}(d x)^{\beta(\tau)}=3 \int \frac{1}{t^{\alpha(\tau)}}(d t)^{\alpha(\tau)}, \\
x(1)=\xi
\end{array}\right.
$$

and for $x>0$, we obtain

$$
\left\{\begin{array}{l}
\int d u=0, \\
u(x, 1)=f(\zeta), \\
\int \frac{1}{x^{\lambda(\tau)}}(d x)^{\lambda(\tau)}=5 \int \frac{1}{t^{\gamma(\tau)}}(d t)^{\gamma(\tau)}, \\
x(1)=\zeta .
\end{array}\right.
$$

The integration of (4.39) and (4.40), by formula (2.15), for $x<0$ leads to

$$
\left\{\begin{array}{l}
u=C_{1}, \\
u(x, 1)=f(\xi), \\
\ln x^{\beta(\tau)}=3 \ln t^{\alpha(\tau)}+C_{2}, \\
x(1)=\xi,
\end{array}\right.
$$


and for $x>0$, it leads to

$$
\left\{\begin{array}{l}
u=C_{3}, \\
u(x, 1)=f(\zeta) \\
\ln x^{\lambda(\tau)}=5 \ln t^{\gamma(\tau)}+C_{4} \\
x(1)=\zeta
\end{array}\right.
$$

From equations (4.41) and (4.42), we obtain the following parametric solution:

$$
\begin{cases}u=f(\xi), & x<0 \\ u=f(\zeta), & x>0 \\ \ln x^{\beta(\tau)}=3 \ln t^{\alpha(\tau)}+\ln \xi^{\beta(\tau)}, & x<0 \\ \ln x^{\lambda(\tau)}=5 \ln t^{\gamma(\tau)}+\ln \zeta^{\lambda(\tau)}, & x>0\end{cases}
$$

which simplifies to

$$
\begin{cases}u=f(\xi), & x<0 \\ u=f(\zeta), & x>0 \\ x^{\beta(\tau)}=\xi^{\beta(\tau)} t^{3 \alpha(\tau)}, & x<0 \\ x^{\lambda(\tau)}=\zeta^{\lambda(\tau)} t^{5 \gamma(\tau)}, & x>0\end{cases}
$$

Let us assume the initial condition $f(x)=\cos x+3 \sin x$, then the solution is

$$
\left\{\begin{aligned}
u(x, t) & =f\left(\left(x^{\beta(\tau)} t^{-3 \alpha(\tau)}\right)^{1 / \beta(\tau)}\right) \\
& =\cos \left(x^{\beta(\tau)} t^{-3 \alpha(\tau)}\right)^{3 / \beta(\tau)}+3 \sin \left(x^{\beta(\tau)} t^{-3 \alpha(\tau)}\right)^{1 / \beta(\tau)}, \quad x<0, \\
u(x, t) & =f\left(\left(x^{\lambda(\tau)} t^{-5 \gamma(\tau)}\right)^{1 / \beta(\tau)}\right) \\
& =\cos \left(x^{\lambda(\tau)} t^{-5 \gamma(\tau)}\right)^{3 / \lambda(\tau)}+3 \sin \left(x^{\lambda(\tau)} t^{-5 \gamma(\tau)}\right)^{1 / \lambda(\tau)}, \quad x>0 .
\end{aligned}\right.
$$

Benchmark 3 (For Example 3) We make sure that (4.44) is the solution, and satisfies (4.36). Differentiating the first and second equation of (4.44) with respect to $x$ with an order of $\beta(\tau)$ and $\gamma(\tau)$, and with respect to $t$ with an order of $\alpha(\tau)$ and $\lambda(\tau)$ gives

$$
\begin{cases}D_{x}^{\beta(\tau)} u(x, t)=D_{x}^{\beta(\tau)} f(\xi), & x<0, \\ D_{t}^{\alpha(\tau)} u(x, t)=D_{t}^{\alpha(\tau)} f(\xi), & x<0, \\ D_{x}^{\lambda(\tau)} u(x, t)=D_{x}^{\lambda(\tau)} f(\xi), & x>0 \\ D_{t}^{\gamma(\tau)} u(x, t)=D_{t}^{\gamma(\tau)} f(\xi), & x>0\end{cases}
$$

leading to

$$
\begin{cases}\frac{\partial^{\beta(\tau)} u}{\partial \partial^{\beta(\tau)}}=f_{\xi}(\xi) \xi_{x}^{[\beta(\tau)]}, & x<0, \\ \frac{\partial^{\alpha(\tau) u}}{\partial \partial^{\alpha(\tau)}}=f_{\xi}(\xi) \xi_{t}^{[\alpha(\tau)]}, & x<0 \\ \frac{\partial^{\lambda(\tau) u}}{\partial x^{\lambda(\tau)}}=f_{\zeta}(\zeta) \zeta_{x}^{[\lambda(\tau)]}, & x>0 \\ \frac{\partial^{\gamma(\tau) u}}{\partial t^{\gamma(\tau)}}=f_{\zeta}(\zeta) \zeta_{t}^{[\gamma(\tau)]}, & x>0 .\end{cases}
$$


Also, we differentiate the thirdand fourth equation of (4.6) with respect to $x$ with an order of $\beta(\tau)$ and $\gamma(\tau)$, and with respect to $t$ with an order of $\alpha(\tau)$ and $\lambda(\tau)$ to get

$$
\begin{cases}D_{x}^{\beta(\tau)} \ln x^{\beta(\tau)}=D_{x}^{\beta(\tau)}\left[3 \ln t^{\alpha(\tau)}+\ln \xi^{\beta(\tau)}\right], & x<0, \\ D_{t}^{\alpha(\tau)} \ln x^{\beta(\tau)}=D_{t}^{\alpha(\tau)}\left[3 \ln t^{\alpha(\tau)}+\ln \xi^{\beta(\tau)}\right], & x<0, \\ D_{x}^{\lambda(\tau)} \ln x^{\lambda(\tau)}=D_{x}^{\lambda(\tau)}\left[5 \ln t^{\gamma(\tau)}+\ln \zeta^{\lambda(\tau)}\right], & x>0, \\ D_{t}^{\gamma(\tau)} \ln x^{\lambda(\tau)}=D_{t}^{\gamma(\tau)}\left[5 \ln t^{\gamma(\tau)}+\ln \zeta^{\lambda(\tau)}\right], & x>0\end{cases}
$$

which, using formula (2.10), leads to

$$
\begin{cases}\frac{\Gamma(1+\beta(\tau))}{x^{\beta(\tau)}}=\frac{\beta(\tau) \xi^{\beta(\tau)-1}}{\xi^{\beta(\tau)}} \xi_{x}^{[\alpha(\tau)]}, & x<0, \\ 0=\frac{3 \Gamma(1+\alpha(\tau))}{t^{\alpha(\tau)}}+\frac{\beta(\tau) \xi^{\beta(\tau)-1}}{\xi^{\beta(\tau)}} \xi_{t}^{[\alpha(\tau)]}, & x<0, \\ \frac{\Gamma(1+\lambda(\tau))}{x^{\lambda(\tau)}}=\frac{\lambda(\tau) \zeta^{\lambda(\tau)-1}}{\zeta^{\lambda(\tau)} \zeta_{x}^{[\lambda(\tau)]},} & x>0, \\ 0=\frac{5 \Gamma(1+\gamma(\tau))}{t^{\gamma(\tau)}}+\frac{\lambda(\tau) \zeta^{\lambda(\tau)-1}}{\zeta^{\lambda(\tau)}} \zeta_{t}^{[\gamma(\tau)]}, & x>0 .\end{cases}
$$

Obtaining $\xi_{x}^{[\beta(\tau)]}, \xi_{t}^{[\alpha(\tau)]}, \zeta_{x}^{[\lambda(\tau)]}$, and $\zeta_{t}^{[\gamma(\tau)]}$ from (4.13), namely

$$
\begin{cases}\xi_{x}^{[\beta(\tau)]}=\frac{\Gamma(1+\beta(\tau)) \xi}{x^{\beta(\tau)} \beta(\tau)}, & x<0 \\ \xi_{t}^{[\alpha(\tau)]}=-\frac{3 \Gamma(1+\alpha(\tau)) \xi}{t^{\alpha(\tau)} \beta(\tau)}, & x<0 \\ \zeta_{x}^{[\lambda(\tau)]}=\frac{\Gamma(1+\lambda(\tau)) \zeta}{t^{\gamma(\tau) \lambda(\tau)}}, & x>0, \\ \zeta_{t}^{[\gamma(\tau)]}=-\frac{5 \Gamma(1+\gamma(\tau)) \zeta}{t^{\gamma(\tau) \lambda(\tau)}}, & x>0,\end{cases}
$$

and substituting (4.50) into (4.47), we have

$$
\begin{cases}\frac{\partial^{\beta(\tau)} u}{\partial x^{\beta(\tau)}}=f_{\xi}(\xi) \frac{\Gamma(1+\beta(\tau)) \xi}{x^{\beta(\tau)} \beta(\tau)}, & x<0, \\ \frac{\partial^{\alpha(\tau)} u}{\partial t^{\alpha(\tau)}}=-f_{\xi}(\xi) \frac{3 \Gamma(1+\alpha(\tau)) \xi}{t^{\alpha(\tau)} \beta(\tau)}, & x<0, \\ \frac{\partial^{\lambda(\tau)} u}{\partial x^{\lambda(\tau)}}=f_{\zeta}(\zeta) \frac{\Gamma(1+\lambda(\tau)) \zeta}{t^{\gamma(\tau) \lambda(\tau)}}, & x>0, \\ \frac{\partial^{\gamma(\tau)} u}{\partial t^{\gamma(\tau)}}=-f_{\zeta}(\zeta) \frac{5 \Gamma(1+\gamma(\tau)) \zeta}{t^{\gamma(\tau)} \lambda(\tau)}, & x>0 .\end{cases}
$$

Substituting (4.51) into (4.36), we have

$$
\begin{cases}-\frac{t^{\alpha(\tau)}}{\Gamma(1+\alpha(\tau))} f_{\xi}(\xi) \frac{3 \Gamma(1+\alpha(\tau)) \xi}{t^{\alpha(\tau)} \beta(\tau)}+\frac{3 x^{\beta(\tau)}}{\Gamma(1+\beta(\tau))} f_{\xi}(\xi) \frac{\Gamma(1+\beta(\tau)) \xi}{x^{\beta(\tau)} \beta(\tau)}=0, & x<0, \\ -\frac{t^{\gamma(\tau)}}{\Gamma(1+\gamma(\tau))} f_{\zeta}(\zeta) \frac{5 \Gamma(1+\gamma(\tau)) \zeta}{t^{\gamma(\tau)} \lambda(\tau)}+\frac{5 x^{\lambda(\tau)}}{\Gamma(1+\lambda(\tau))} f_{\zeta}(\zeta) \frac{\Gamma(1+\lambda(\tau)) \zeta}{t^{\gamma(\tau) \lambda(\tau)}}=0, & x>0 .\end{cases}
$$

Therefore (4.6) is the solution of (4.2). The answer (4.6) also satisfies the initial condition at $t=0$, since $x=\xi$ for $x<0$ and $x=\zeta$ for $x>0$.

Remark 5 The classic form of Example 3 where $\alpha(\tau)=\beta(\tau)=\gamma(\tau)=\lambda(\tau)=1$ is

$$
\left\{\begin{array}{ll}
t \frac{\partial u}{\partial t}+3 x \frac{\partial u}{\partial x}=0, & x<0, \\
t \frac{\partial u}{\partial t}+5 x \frac{\partial u}{\partial x}=0, & x>0,
\end{array}, \quad u(x, 0)=f(x)=\cos x+3 \sin x\right.
$$



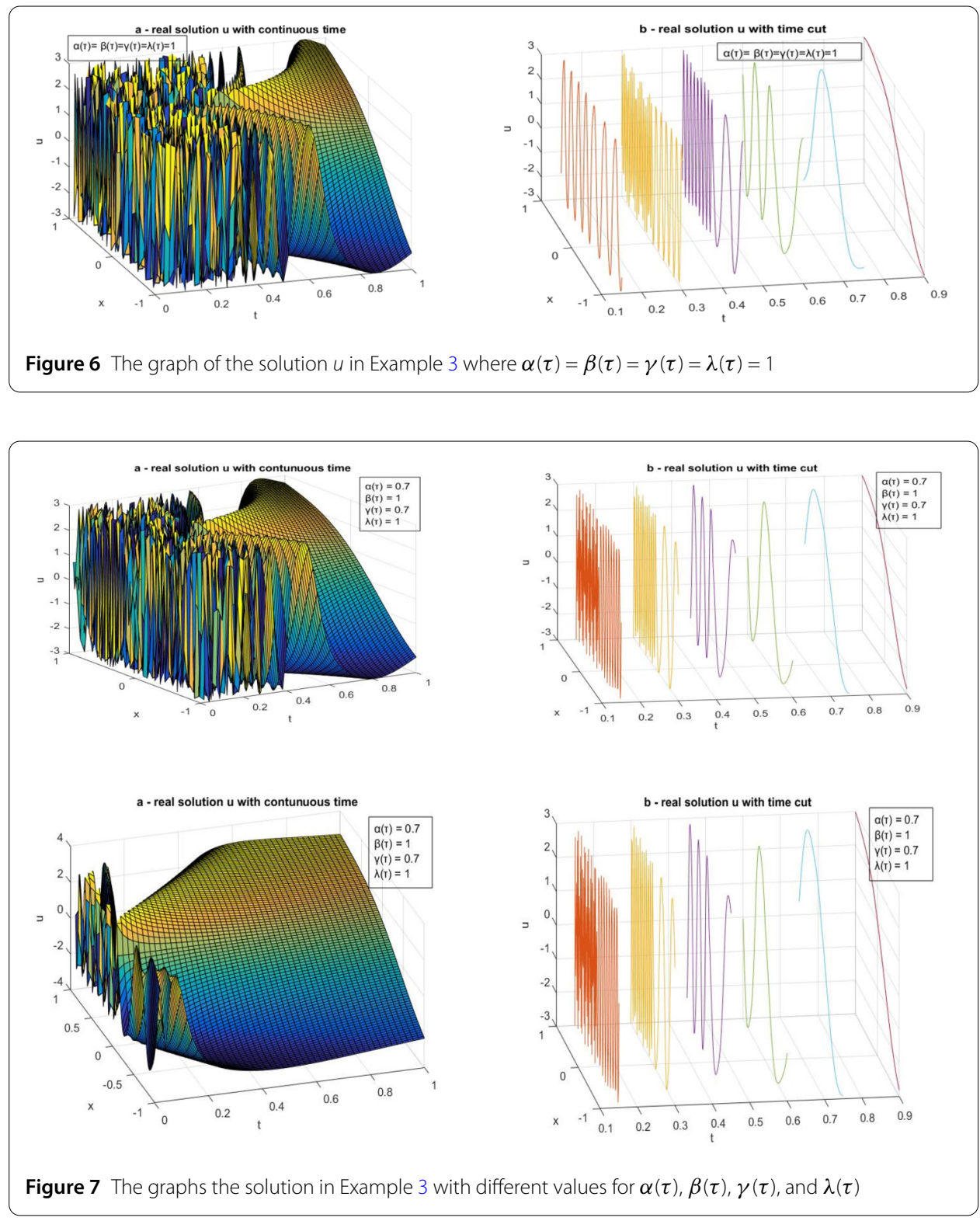

therefore, the solution is

$$
\begin{cases}u(x, t)=f\left(x t^{-3}\right)=\cos \left(x t^{-3}\right)^{3}+3 \sin x t^{-3}, & x<0 \\ u(x, t)=f\left(x t^{-3}\right)=\cos \left(x t^{-3}\right)^{3}+3 \sin x t^{-5}, & x>0 .\end{cases}
$$

Its graph is given in Fig. 6.

The graphs of solution $u$ with different values for $\alpha(\tau), \beta(\tau), \gamma(\tau)$ and $\lambda(\tau)$ are given in Fig. 7.

\section{Summary}

We have proved the existence and uniqueness of the interface coupled arbitrary-order fractional hyperbolic nonlinear scalar conservation law under some conditions. We have 


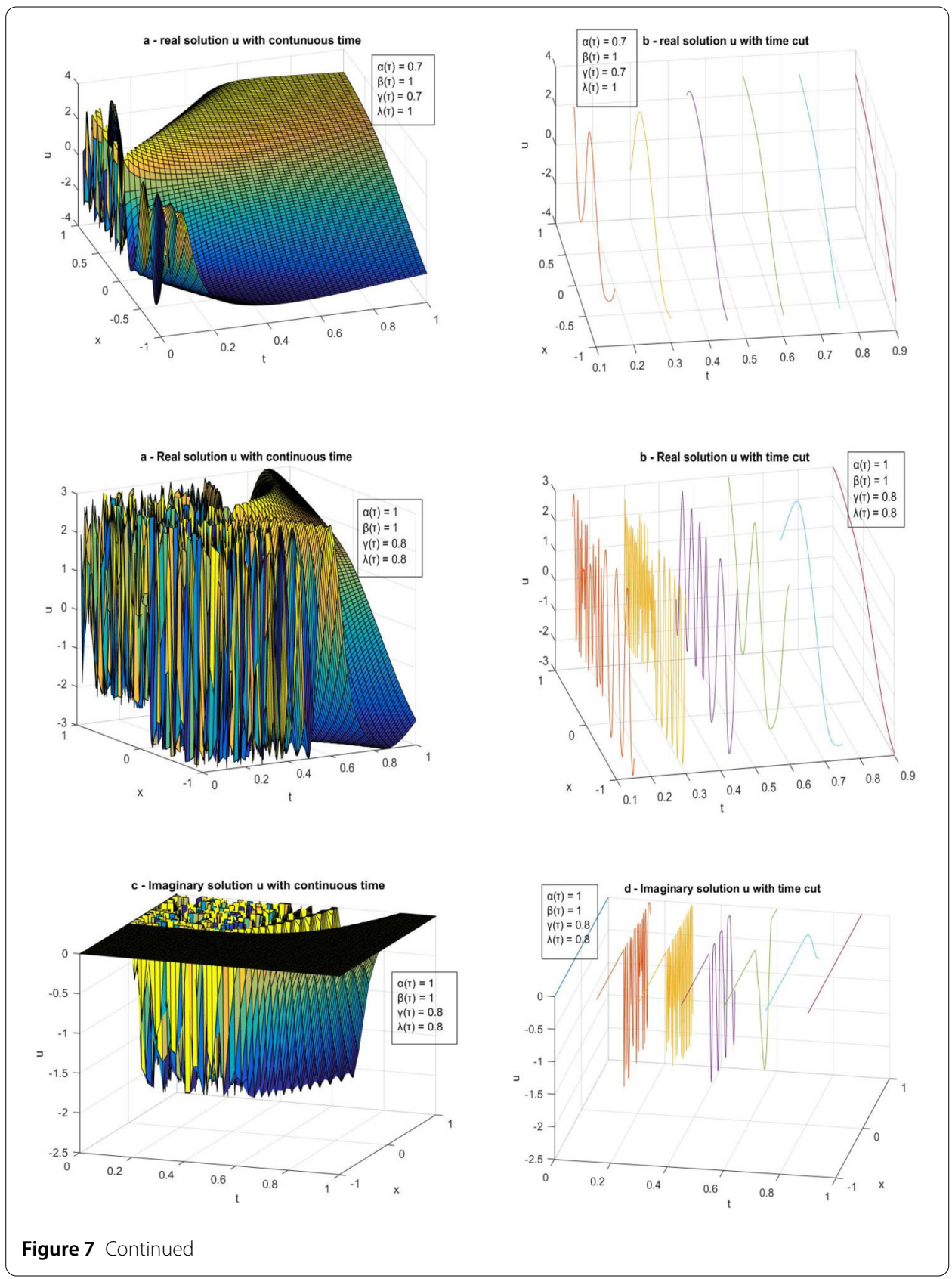

used the generalization of the classical characteristic method that is extended to the fractional characteristic method. Further, we used the generalization of some formulae from the integer-order calculus to the constant-order and arbitrary-order fractional calculus. In the process of proving the existence and the uniqueness of IC AOF HNSCL, we have developed an analytical method that can be used to solve FPDE problems. The feature of this technique is the ability to show that the obtained solutions satisfy the FPDE, so it can be used as a benchmark in the problems to ensure that the results are correct and exact. And finally, we've shown the application of this approach by presenting a few physical examples. In addition, we've also shown the graphs for the different values of $\alpha(\tau), \beta(\tau), \gamma(\tau)$ and $\lambda(\tau)$ in each problem and also provided the benchmark as well. 


\section{Acknowledgements}

Reza Shirkhorshidi would like to express his sincere gratitude and appreciation to Fatemeh Zahedifar, who helped revised the English grammar of the paper.

Funding

This paper is support by the University of Malaya. The grant number is IIRG001C-2019.

\section{Abbreviations}

IC,Interface Coupling; TS, Time and Spatial; LC, Liouville-Caputo; VOF, Variable-Order Fractional; AOF, Arbitrary-Order Fractional; HCL, Hyperbolic Conservation Laws; NHSCL, Nonlinear Hyperbolic Scalar Conservation Laws; AFCM, Arbitrary Fractional Characteristic Method; PDEs, Partial Differential Equations; ODEs, Ordinary Differential Equations; FC, Fractional Calculus; FD, Fractional Derivative; FI, Fractional Integral; DEs, Differential Equations; FODEs, Fractional Ordinary Differential Equations; FODE, Fractional Ordinary Differential Equation; FPDEs, Fractional Partial Differential Equations; FPDE, Fractional Partial Differential Equation.

\section{Availability of data and materials}

Not applicable.

\section{Competing interests}

The authors declare that they have no known competition for financial interests or personal relationships that could have appeared to influence the work reported in this paper

\section{Authors' contributions}

The main idea of this paper was proposed by RS, WAMO and DR. RS prepared the manuscript initially and performed all the steps of the proofs in this research. All authors read and approved the final manuscript.

\section{Author details}

${ }^{1}$ Institute of Mathematical Sciences, University of Malaya, Kuala Lumpur, Malaysia. ${ }^{2}$ Institute of Mathematical Sciences, Faculty of Science, University of Malaya, Kuala Lumpur, Malaysia. ${ }^{3}$ Department of Mathematics, Imam Khomeini International University, Qazvin, Iran. ${ }^{4}$ Faculty of Computer Science and Information Technology, University of Malaya, Kuala Lumpur, Malaysia.

\section{Publisher's Note}

Springer Nature remains neutral with regard to jurisdictional claims in published maps and institutional affiliations.

Received: 10 July 2020 Accepted: 25 October 2020 Published online: 20 November 2020

\section{References}

1. Myint-U, T., Debnath, L.: Linear Partial Differential Equations for Scientists and Engineers (2007)

2. Wu, G.C.: A fractional characteristic method for solving fractional partial differential equations. Appl. Math. Lett. 24, 1046-1050 (2011)

3. Mirzazadeh, M., et al.: Optical solitons in nonlinear directional couplers by sine-cosine function method and Bernoulli's equation approach. Nonlinear Dyn. 81, 1933-1949 (2015)

4. Eslami, M., Mirzazadeh, M.: Optical solitons with Biswas-Milovic equation for power law and dual-power law nonlinearities. Nonlinear Dyn. 83, 731-738 (2016)

5. Mirzazadeh, M., Eslami, M., Biswas, A.: Dispersive optical solitons by Kudryashov's method. Optik 125, 6874-6880 (2014)

6. Nazarzadeh, A., Eslami, M., Mirzazadeh, M.: Exact solutions of some nonlinear partial differential equations using functional variable method. Pramana J. Phys. 81, 225-236 (2013)

7. Biswas, A., Mirzazadeh, M., Eslami, M.: Dispersive dark optical soliton with Schödinger-Hirota equation by G'/G-expansion approach in power law medium. Optik 125, 4215-4218 (2014)

8. Eslami, M., Neyrame, A., Ebrahimi, M.: Explicit solutions of nonlinear ( $2+1)$-dimensional dispersive long wave equation. J. King Saud Univ., Sci. 24, 69-71 (2012)

9. Liu, J.G., Eslami, M., Rezazadeh, H., Mirzazadeh, M.: Rational solutions and lump solutions to a non-isospectral and generalized variable-coefficient Kadomtsev-Petviashvili equation. Nonlinear Dyn. 95, 1027-1033 (2019)

10. Mirzazadeh, M., Eslami, M.: Exact solutions of the Kudryashov-Sinelshchikov equation and nonlinear telegraph equation via the first integral method. Nonlinear Anal., Model. Control 17, 481-488 (2012)

11. Baleanu, D., Agrawal, O.P., Muslih, S.I.: Lagrangians with linear velocities within Hilfer fractional derivative. In: Proceedings of the ASME Design Engineering Technical Conference, vol. 3, pp. 335-338 (2011)

12. Tenreiro Machado, J.A.: Fractional derivatives: probability interpretation and frequency response of rational approximations. Commun. Nonlinear Sci. Numer. Simul. 14, 3492-3497 (2009)

13. Machado, J.A.T.: And I say to myself: what a fractional world! Fract. Calc. Appl. Anal. 14, 635-654 (2011)

14. Magin, R., Ortigueira, M.D., Podlubny, I., Trujillo, J.: On the fractional signals and systems. Signal Process. 91, 350-371 (2011)

15. Baleanu, D., Shiri, B., Srivastava, H.M., Al Qurashi, M.: A Chebyshev spectral method based on operational matrix for fractional differential equations involving non-singular Mittag-Leffler kernel. Adv. Differ. Equ. 2018, 353 (2018)

16. Baleanu, D., Jajarmi, A., Sajjadi, S.S., Mozyrska, D.: A new fractional model and optimal control of a tumor-immune surveillance with non-singular derivative operator. Chaos 29, 083127 (2019)

17. Angelini, F., Herzel, S.: Journal of computational and applied. J. Comput. Appl. Math. 259, 385-393 (2014)

18. Podlubny, I. Fractional differential equations to methods of their solution and some of their applications. In: Fractional Differential Equations: An Introduction to Fractional Derivatives 340. Academic Press, San Diego (1998) 
19. Kilbas, A.A., Srivastava, H.M., Trujillo, J.J.: Theory and Applications of Fractional Differential Equations. Elsevier, Amsterdam (2006)

20. Samko, S.G., Ross, B.: Integration and differentiation to a variable fractional order. Integral Transforms Spec. Funct. 1, 277-300 (1993)

21. Lorenzo, C.F., Hartley, T.T.: Initialization, conceptualization, and application in the generalized (fractional) calculus. Crit. Rev. Biomed. Eng. 35, 447-553 (2007)

22. Coimbra, C.F.M.: Mechanics with variable-order differential operators. Ann. Phys. 12, 692-703 (2003)

23. Chechkin, A.V., Gorenflo, R., Sokolov, I.M.: Fractional diffusion in inhomogeneous media. J. Phys. A, Math. Gen. 38(42), L679-L684 (2005)

24. Sun, H., Chen, W., Chen, Y.: Variable-order fractional differential operators in anomalous diffusion modeling. Phys. A, Stat. Mech. Appl. 388(21), 4586-4592 (2009)

25. Diaz, G., Coimbra, C.F.M.: Nonlinear dynamics and control of a variable order oscillator with application to the van der Pol equation. Nonlinear Dyn. 56, 145-157 (2009)

26. Chang, A., Sun, H.: Time-space fractional derivative models for $\mathrm{CO}_{2}$ transport in heterogeneous media. Fract. Calc. Appl. Anal. 21,151-173 (2018)

27. Wu, G.C., Deng, Z.G., Baleanu, D., Zeng, D.Q.: New variable-order fractional chaotic systems for fast image encryption. Chaos 29, 083103 (2019)

28. Obembe, A.D., Hossain, M.E., Abu-Khamsin, S.A.: Variable-order derivative time fractional diffusion model for heterogeneous porous media. J. Pet. Sci. Eng. 152, 391-405 (2017)

29. Cai, W., Chen, W., Fang, J., Holm, S.: A survey on fractional derivative modeling of power-law frequency-dependent viscous dissipative and scattering attenuation in acoustic wave propagation. Appl. Mech. Rev. 70(3), 030802 (2018)

30. Yan, Y., Sun, Z.Z., Zhang, J.: Fast evaluation of the Caputo fractional derivative and its applications to fractional diffusion equations: a second-order scheme. Commun. Comput. Phys. 22, 1028-1048 (2017)

31. Langlands, T.A.M., Henry, B.I.: Fractional chemotaxis diffusion equations. Phys. Rev. E, Stat. Nonlinear Soft Matter Phys. 81(5), $051102(2010)$

32. Povstenko, Y., Klekot, J.: The Dirichlet problem for the time-fractional advection-diffusion equation in a line segment. Bound. Value Probl. 2016, 89 (2016)

33. Zhang, L., Li, S.: Regularity of weak solutions of the Cauchy problem to a fractional porous medium equation. Bound. Value Probl. 2015, 28 (2015)

34. Ren, Y., Tao, M., Dong, H., Yang, H.: Analytical research of $(3+1)$-dimensional Rossby waves with dissipation effect in cylindrical coordinate based on Lie symmetry approach. Adv. Differ. Equ. 2019, 13 (2019)

35. Abdelkawy, M.A., Zaky, M.A., Bhrawy, A.H., Baleanu, D.: Numerical simulation of time variable fractional order mobile-immobile advection-dispersion model. Rom. Rep. Phys. 67, 773-791 (2015)

36. Gupta, P.K., Singh, M.: Homotopy perturbation method for fractional Fornberg-Whitham equation. Comput. Math Appl. 61, 250-254 (2011)

37. Saha Ray, S.: Analytical solution for the space fractional diffusion equation by two-step Adomian decomposition method. Commun. Nonlinear Sci. Numer. Simul. 14, 1295-1306 (2009)

38. Nazari, D., Shahmorad, S.: Application of the fractional differential transform method to fractional-order integro-differential equations with nonlocal boundary conditions. J. Comput. Appl. Math. 234, 883-891 (2010)

39. Cui, Z., Mao, Z., Yang, S., Yu, P.: Approximate analytical solutions of fractional perturbed diffusion equation by reduced differential transform method and the homotopy perturbation method. Math. Probl. Eng. 2013, Article ID 186934 (2013)

40. Abdel-Salam, E.A.B., Yousif, E.A.: Solution of nonlinear space-time fractional differential equations using the fractional Riccati expansion method. Math. Probl. Eng. 2013, Article ID 846283 (2013)

41. Zhang, S., Zhang, H.Q.: Fractional sub-equation method and its applications to nonlinear fractional PDEs. Phys. Lett. A 375, 1069-1073 (2011)

42. Abdelkawy, M.A., El-Kalaawy, O.H., Al-Denari, R.B., Biswas, A.: Application of fractional sub-equation method to nonlinear evolution equations. Nonlinear Anal., Model. Control 23, 710-723 (2018)

43. Gepreel, K.A., Mohamed, M.S.: Analytical approximate solution for nonlinear space-time fractional Klein-Gordon equation. Chin. Phys. B 22, 1 (2013)

44. Yulita, R., Batiha, B., Shatnawi, M.T.: Solutions of fractional Zakharov-Kuznetsov equations by fractional complex transform. Int. J. Appl. Math. Res. 5, 24-28 (2016)

45. Shirkhorshidi, S.M.R., Rostamy, D., Othman, W.A.M., Awang, M.A.O.: The arbitrary-order fractional hyperbolic nonlinear scalar conservation law. Adv. Differ. Equ. 2020, 1 (2020)

46. Tavares, D., Almeida, R., Torres, D.F.M.: Caputo derivatives of fractional variable order: numerical approximations, Commun. Nonlinear Sci. Numer. Simul. 35, 69-87 (2016)

47. Jumarie, G.: On the derivative chain-rules in fractional calculus via fractional difference and their application to systems modelling. Cent. Eur. J. Phys. 11(6), 617-633 (2013)

48. Wu, G.: A fractional characteristic method for solving fractional partial differential equations. Appl. Math. Lett. 24(7), 1046-1050 (2011)

49. Godlewski, E., Raviart, P.A.: The numerical interface coupling of nonlinear hyperbolic systems of conservation laws: I. The scalar case. Numer. Math. 97, 81-130 (2004) 